\title{
Characterization of capillary waves: a review and a new optical method
}

\author{
Radomir I. Slavchov ${ }^{1}$, Boyan Peychev ${ }^{1}$, A. Said Ismail ${ }^{1 *}$ \\ ${ }^{1}$ School of Engineering and Materials Science, Queen Mary University of London, \\ London E1 4NS, United Kingdom
}

Corresponding author: a.ismail@qmul.ac.uk

Keywords: capillary waves, ripples, surface elasticity, dispersion equation, decay length, high-speed video camera

\begin{abstract}
The methods to study capillary waves have been reviewed, together with the emerging practical applications of theirs and new theoretical developments in the field. The focus is on monochromatic ripples of frequency in the range $0.1-10 \mathrm{kHz}$. A capillary wave apparatus has been constructed that combines several recent advances on the technique. It is based on profilometry of waves decaying with distance, with a highspeed video camera detecting light refracted by the surface. A code to process the images has been developed that executes a regression analysis to determine the characteristics of the wave. High precision and accuracy have been achieved: standard deviation from the mean of $\pm 0.5 \%$ for the wavelength and $\pm 7 \%$ for the decay length; mean deviations from the theoretical values $\pm 0.2 \%$ for the wavelength and $\pm 5 \%$ for the decay length. An analytic approximation for the dispersion relation has been used to determine the Gibbs elasticity of a surfactant monolayer from the data for decay length vs. frequency. The elasticity of an octanol monolayer has been determined with precision of $\pm 1 \mathrm{mN} / \mathrm{m}$, in excellent agreement with the theoretical value. Surface tension can be measured from the wavelength data with precision of $\pm 0.3 \mathrm{mN} / \mathrm{m}$. It has been demonstrated that the effect of the surface elasticity on the wavelength is significant and accurate wavelength data can actually be used to determine the elasticity if the surface tension is known.
\end{abstract}




\section{Introduction}

The ripples at a liquid interface carry rich information about the properties of the interface and the processes taking place there. The wavelength $\lambda$ can be used to infer the surface tension $\sigma$ [1], while the decay length $L_{\mathrm{d}}$ and the decay time $t_{\mathrm{d}}$ are controlled by the viscosity of the liquid [2], the Gibbs elasticity $E$ of the adsorbed surfactant monolayer [3], the diffusion- [3] or barrier-controlled [4] adsorption-desorption kinetics, and the related [5] surface viscosity. Numerous studies have demonstrated the potential of the liquid-surface relaxational spectrometry [4] - i.e. the surface wave apparatus - as a tool for characterization of interfaces. The method is quick and, at least in theory, could be very precise. Its main use currently is for characterization of liquid interfaces, including surfactant monolayers $[4,6]$, polymer layers [6,7], electrolyte solutions [8], sea water [9], oil films [10] etc. The technique is excellent for measuring ultralow interfacial tensions [11,12] and to detect surface phase transitions $[13,14]$. Capillary wave apparatuses have been utilized as contactless tenisometers-viscosimeters for surfactant-free liquids $[15,16,17]$. Practical applications of these tools are also beginning to emerge. A surface relaxational spectrometer was used in a sensor for exhaled pulmonary surfactant [18]. Spherical waves on inkjet droplets are being used to study the kinetics of adsorption right after jet break-up, and were claimed to carry information about the variation of the viscosity of the droplet with time [19,20,21]. Similarly, the growth of waves on the surface of a jet before break-up has been used to determine the surface tension of the jet [22].

The capillary waves are responsible for several mechanisms of destabilization of jets, films and droplets: examples are the jet break-up in continuous inkjet printing [23]; bubble entrapment and gas filament break-up [24]; ripples break liquid films via the Scheludko-Vrij mechanism $[25,26,27]$ etc. As a result, the fundamental understanding of the wave growth is essential for the control of the destabilization of multi-phase systems. The growth rate of waves at the surface of an electrified jet can be used to estimate the break-up length [28] and understand the transition from varicose to whipping instabilities in electrohydrodynamic jetting [29]. The ripples play a role in a number of surface phenomena, sometimes in a nonintuitive way. For example, the capillary waves in thin films and membranes contribute significantly to the surface forces [30]. Near the critical point, they contribute also to the value of the interfacial tension [31]. Thermal capillary waves have been hypothesized to control the propagation of the three-phase contact line over heterogeneous solids [32]. A small body (such as an atomic force microscope tip) that interacts with the surface of a liquid experiences wave resistance, i.e. it generates energy-dissipating capillary waves [33].

Unfortunately, the surface wave spectrometry has not found widespread use, despite the obvious value and the continuous efforts since the 1930s. Currently, ripple tanks are widely available for educational purposes, but no commercial scientific apparatus exists [6]. The reason are a number of technical complications (discussed, e.g., in ref. [6,34]) and perhaps also the fact that the interpretation of the data is not straightforward.

Here, we review the current state of the art of the methods used to study ripples, the experimental difficulties, and some recent theoretical advances in the field. We then proceed to show how widely available laboratory equipment - loud speaker and high-speed video camera - can be used to generate and characterize capillary waves with high precision, comparable and even superior to most data reported in the literature. 


\section{Experimental techniques}
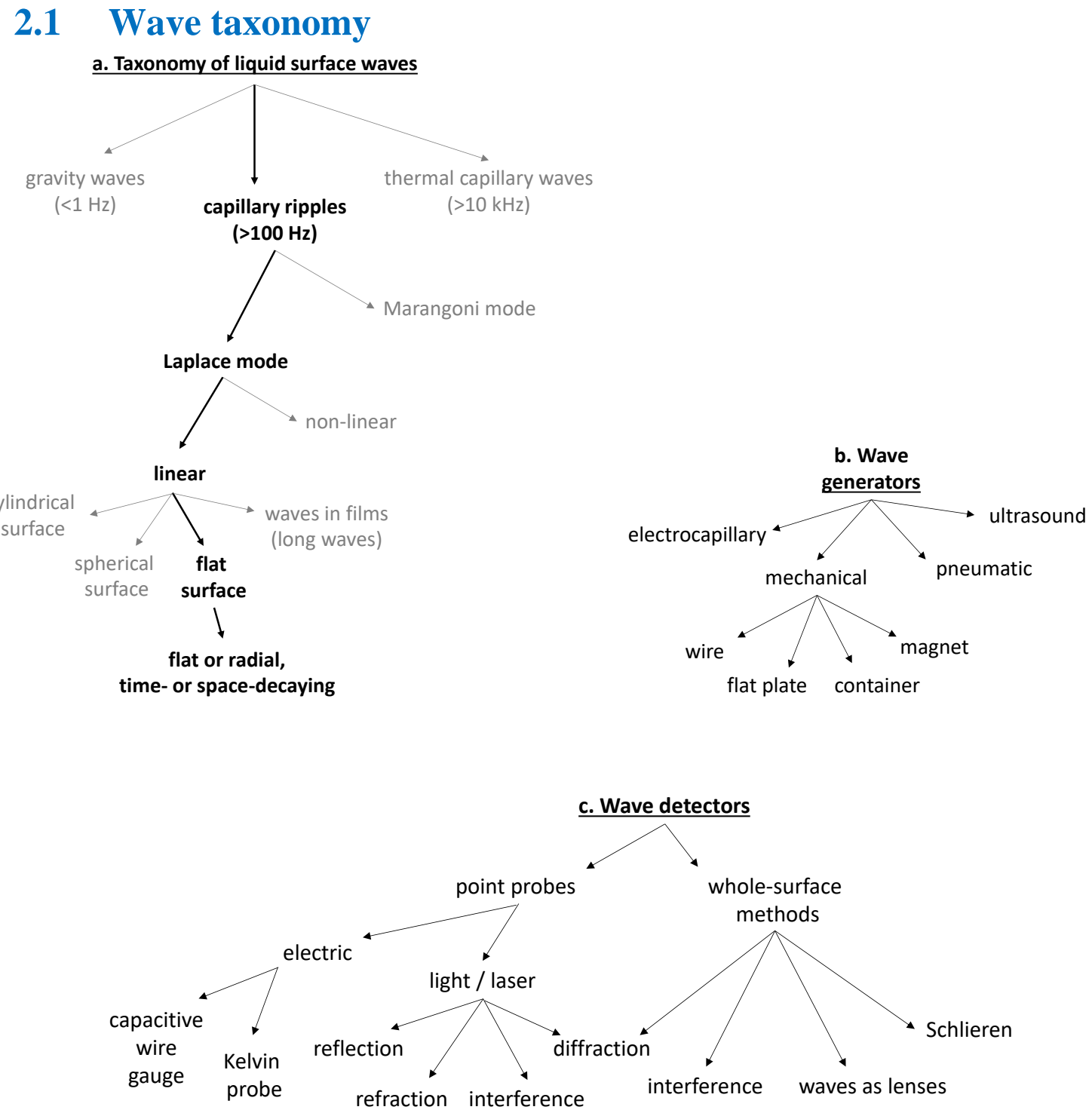

Figure 1a. Surface wave taxonomy.

$\mathrm{b}$ and $\mathrm{c}$. Methods to generate and detect the properties of a wave.

A variety of waves exist at the interface between two fluids (Figure 1a). Waves of different frequency are controlled by different factors. Our focus here are the ripples, i.e. capillary waves of wavelength of the order of $\sim 0.1-1 \mathrm{~mm}$ and frequency $\sim 10^{2}-10^{4} \mathrm{~Hz}$. Waves of wavelength $\lambda$ approaching or exceeding the capillary length $(\sigma / \Delta \rho g)^{1 / 2}$ are affected by gravity, and one speaks of capillary-gravity waves $(1-10 \mathrm{~mm}, 10-100 \mathrm{~Hz}$ for water), or pure gravity waves $(\lambda>1 \mathrm{~cm}$, $v<10 \mathrm{~Hz}$ ). The study of capillary-gravity waves has a long history in relation to sea science $[35,9,10]$. On the other end of the spectrum are the thermal capillary waves, of frequency in the range $10^{4}-10^{6} \mathrm{~Hz}$. This range is accessible with light scattering and X-ray reflectivity; the results from these techniques have been recently reviewed in detail by Langevin [6].

At the surface of single-component Newtonian liquids, the Navier-Stokes and Laplace equations produce a wave dispersion condition that has a single solution, corresponding to a weakly-decaying transverse wave. In contrast, for interfaces of inherent surface elasticity (such as those covered with a surfactant layer, the surface of a liquid mixture etc.), the wave dispersion 
condition has two physical solutions [5] - i.e., ripples exist in two wave modes. One is the regular transverse (Laplace) mode; the other is longitudinal and fast-decaying (Marangoni or Lucassen) mode [36]. The Laplace and the Marangoni waves of the same frequency are of slightly different wavelength, very different decay length, and different depth of protrusion into the substrate. The two modes are studied using two respective types of capillary wave apparatuses. The decay length of the Laplace mode varies strongly with the surface elasticity, but at $E>1-3 \sigma$, the surface becomes completely tangentially immobile and the dynamic surface characteristics cease to have an effect on the wave [4,3]. The Marangoni mode is more sensitive to the viscoelastic properties of the surface at high $E$, but is more difficult to study $[37,38,39]$. Our focus here are the transverse Laplace waves.

The next distinction is the one between linear and non-linear capillary waves. When the amplitude $a$ is comparable with the wavelength $\lambda$, non-linear effects appear, due to the convective term in the Navier-Stokes equation, the non-linear nature of the Laplace boundary condition, and the non-linear surface equation of state of the surfactant monolayer. The respective "turbulent" waves are a whole separate field of study that has been recently reviewed by Falcon and Mordant [40]. Among the major achievements of the theory of the turbulent waves are the explanation of the spectrum of the sea waves, the modelling of the resonant interactions between waves, and the discovery of several mechanisms of wave energy dissipation. Non-linear waves will not be considered here, but the experimental methods used to study them will be discussed, as they are applicable also to linear waves.

Waves in geometrically confined systems differ significantly from those at a flat surface [41]. Particularly important are the waves on a cylindrical jet, in view of their role in inkjet printing [23]. Waves on droplets are another important example that has many applications [19,20,21]; confinement brings about new effects also in waves in liquid films $[27,30,42]$.

The linear waves on a flat surface can have different geometry depending on the source (propagating or standing, flat or radial waves). The nature of the source controls also the spacetime decay of the waves. A stationary source of fixed power, after a short non-steady state period, will produce waves that decay with space but of time-independent amplitude. A short disturbance of the surface will generate waves decaying also with time. The apparatus we discuss in sec. 4 below is designed for investigation of flat Laplace ripples decaying with space.

\subsection{Techniques to generate waves}

An apparatus for characterization of ripples requires two main elements: a generator of waves and a detector. The most common methods to excite Laplace ripples are mechanical (see Figure 1b). In this case, the surface wave generator consists of an electromagnetic acoustic generator and a transmitting device. The acoustic generators are made of a sinusoidal voltage generator connected to an electrodynamic vibrator, producing frequency in the range of $10-1000 \mathrm{~Hz}[43,44,4]$. The transmitting device is usually a dipped wire or plate [43], a glass capillary [4] or a plastic cylinder [44] connected to the acoustic generator. The container can also be used as the transmitting device [12,45,46,47,48]. Chantelot et al. [49] used a floating magnet driven by an electromagnetic actuator instead.

The advantages of the mechanical generators are simplicity of construction, the range of options to control the amplitude of the wave (via the power of the acoustic generator, the size of the dipping wire etc.), and most of all, the versatility - with minor modifications, the equipment can be used to create flat, radial, linear and non-linear waves on any interface. Among the disadvantages are that non-linear oscillations appear in the vicinity of the resonance frequencies of the transmitting device (see sec. 4.2 below); the complex role of the contact angle and the meniscus (a meniscus deforms the surface yet it is required for efficient transmission [47]); finally, careful cleaning of the dipping plate or the capillary are necessary. 
To overcome the latter two disadvantages, several contactless generators have been developed. The most common of those is electrocapillary excitation, where oscillating local electric field is used as a transmitter [14,15,16,17,50,51]. The field is created by a metal blade (for flat waves) or by a sharp edge (for radial waves). The electromechanical transmission can produce, in theory, undesired electrocapillary effects disturbing the wave, but the question has not been studied systematically. A new promising contactless method utilizes pneumatic transmission [34], where the wave is excited by a local pressure fluctuations of the gas pillar between a wave guider and the studied surface. In theory, this method can also affect the waves by producing local cooling due to accelerated evaporation, as variations of the temperature with even small part of a degree can cause thermal Marangoni effect [52] disturbing the wave. Another disadvantage is that the pneumatic method is applicable for radial waves at the liquid|air interface only. A third contactless method has been proposed where focussed ultrasound beam is used to generate radial waves at a liquid|liquid interface [11].

There are various options for generating capillary waves on the surface of a jet, which are used to control its break-up [23]. The standard approach is mechanical modulation of the jet with a piezoelectric device. A novel and flexible method is via periodic thermal modulation: a heater is installed in the vicinity of the nozzle, and it produces temperature swings under the action of voltage pulses. These pulses results in a thermal Marangoni effect that excites the waves [23,53]. The same approach is, in theory, applicable also for excitation of waves on a flat surface.

\subsection{Techniques to detect waves}

Point methods for wave characterization. It is common the detection to be in a set of fixed points of the surface. The first class of point methods is based on interaction of the surface with focussed beam of light. The classical approach is to use cathode-ray projection tube as a spot source of light, for viewing waves stroboscopically [43]. The light spot scans past a horizontal slit [43]. Laser probe can be used in a similar manner [15,50], and is currently the most popular detection technique to study linear waves. Optical methods based on absorption, reflection or refraction of laser beams are popular also for non-linear waves [40]. The wavelength can be determined by using the ripples as diffraction grating [54,55]. A laser interferometer has been developed $[16,17]$, where the phase difference between a beam reflected from the liquid surface and a reference beam is used to infer the amplitude of the wave with precision of the order of $5 \mathrm{~nm}$. A similar approach is used by the laser Doppler vibrometer that allows the surface velocity in a point to be determined [56,57]; this method requires the addition of light scattering particles in the studied fluid. Local radiative heating of the surface is possible with some of the optical detection techniques, which can cause Marangoni disruptions of the surface.

Another class of detection methods is via electric probes. Noskov used a capacity wave detector [4], which is a metal plate parallel to the surface of width much smaller than the wavelength. The oscillation of the liquid leads to oscillation in the capacity of the condenser and alternating electric current (a type of a Kelvin probe). A grounded platinum plate in the bulk liquid is used as a reference electrode. Capacitive wire gauges are a common method to study non-linear waves $[58,40]$; the amplitude of the wave in a point is measured by making use of the proportionality between the capacity of a thin wire and the immersion length. Amplitudes smaller than a few $\mu \mathrm{m}$ are difficult to detect with this method, which limits its application to long-wavelength or non-linear waves.

Space-time methods for wave characterization. An alternative approach is to observe the wavy surface as a whole (instead of a few test points). Such space-time measurements are quickly winning ground in the field of non-linear waves, and Falcon and Mordant listed seven methods of this type [40]. For linear ripples, this approach is uncommon and only a few recent studies utilized it. Shmyrov et al. [34] used optical interferometry and spatial phase shifting to 
quantify the profile of a wavy surface with high accuracy, producing a $3 \mathrm{D}$ reconstruction of the entire interface. They obtained the wave characteristics by post-processing of the 3D reconstruction. A much older technique that can be classified as space-time is direct optical imaging of the surface, which is based on the lens effect from the wave crests and troughs on the light crossing the rippled surface [9,59]; this option allows 3D reconstruction under the condition that the surface shape has flat or radial symmetry, which is fulfilled for the ripples normally investigated with capillary waves apparatuses. Moisy et al. [60] reconstructed the surface topography of gravitational-capillary waves $(\sim 10 \mathrm{~Hz})$ using free-surface synthetic Schlieren technique (distortion of a printed pattern by refraction from the deformed liquid surface); a number of variants of this technique exist $[48,49,61,62,63]$ and Vinnichenko et al. [44] used reflection rather than refraction. The variants of the Schlieren imaging are somewhat limited in space resolution and are more suited to study gravity-capillary waves than shorter capillary waves. The use of high-speed video cameras as detection device is becoming more and more common, especially for non-linear gravity and gravity-capillary waves [40,62]; few studies, however, utilize cameras for linear capillary waves. Krutyansky et al. [11] used a side camera to resolve the profile of the wave, but high amplitudes are required in this case which inevitably introduces non-linearity. The use of a combination of cameras allows complete space-time reconstruction of the fluid surface motion (stereoscopy), which was used to analyse non-linear gravity waves [64]. All these apparatuses are essentially profilometers. There are other ways to access the characteristics of the wave. For example, Strickland et al. used fluorescent imaging to obtain a map of the adsorption of a fluorescent lipid [48] (the adsorption wave is conjugated to the mechanical surface wave, see sec. 3 below). Another example is the determination of the velocity of tracer particles, which is often used to characterize non-linear gravity waves [40].

The post-processing of the data is much simpler for the point methods - the amplitude vs. time data can be analysed via fast Fourier transform (if the wave does not decay with time) or via regression (for linear monochromatic decaying wave). The slow and intricate image processing is a drawback of most space-time methods (see, e.g., [67]). The algorithms for 3D image reconstruction are particularly well-developed for the variants of the Schlieren technique $[60,63]$.

All methods for characterization of capillary waves on the surface of drops and jets are space-time. For the characterization of capillary waves on liquid filaments, optical approaches have been used extensively $[68,69,70,71]$. The most common of these techniques is shadowgraphy, where the jet or the droplets are placed between a light source and a camera. For example, Dong et al. [71] used this approach to observe liquid thread break-up caused by the instability of propagating capillary waves. Wang et al. [72] characterized the propagation of capillary waves on images of a liquid thread at various Ohnesorge numbers, liquid thread aspect ratios, and surface perturbation amplitude. In this way, they were able to establish a map of the break-up regimes, and verified a capillary wave superposition model that predicts the fate of the thread. This map facilitates the control of the break-up to avoid formation of satellites in drop-on-demand inkjet printing applications. The satellites are eliminated by increasing the viscosity, as the viscous friction dampens the capillary waves [71]. The oscillation of a liquid thread contracted into a drop has been used to measure the surface tension and - allegedly - the viscosity of the liquid, via the oscillation period and the damping rate $[73,20]$. Similarly, the oscillation of electrified conducting drops surrounded by a dielectric fluid was optically observed by Mohamed et al. [74]. The temporal evolution of the drop apex can actually be used to measure the liquid dynamic properties in a similar manner.

The shadowgraph visualization of capillary waves on jets and droplets can be done with high-speed video camera or a stroboscopic system, in which a strobe illumination is used to visualize periodic events. In the latter system, sequential images are taken with incremental 
trigger delay to observe the phenomena and take measurements with time interval of the order of $10 \mu \mathrm{s}$ [75]. This provides an inexpensive visualizing system which has been used, for instance, to study the role of capillary waves in the break-up regimes of electrified jet [29] and to measure the jet break-up length [28].

The break-up in jets is caused by the Rayleigh-Plateau instability (waves of wavelength longer than the circumference of the jet diverge). The Rayleigh dispersion relation [76] identifies the most unstable wavelength and allows the estimation of the size of drops generated from the jet break-up. Experimental visualization of Gaussian wave packets evolution in cylindrical jets combined with temporal theoretical analysis found that the growth rate of the wave indeed corresponds to the maximum of Rayleigh dispersion relation [77]. Recently, the capillary wave profile on the jet surface was extracted using a laser photodiode pair to study self-stimulated dynamics of jets [78]. This was used to create a feedback loop to control the jet break-up by tuning the inlet pressure perturbations to optimal frequency with minimum stimulation amplitude.

Experimental difficulties. A major problem of the capillary wave characterization techniques is that they frequently produce unreliable results, for no apparent reason. The surface elasticity determined by this method is often inadequate. For example, Wasan et al. [38] found Gibbs elasticity that passes through a maximum and strongly disagrees with the one determined from the surface tension isotherm. Their tentative explanation with phase transition is unlikely - the LE-LC should appear at higher concentrations for the surfactant they studied. Lemaire and Langevin [39] found serious disagreement between the surface tension measured with capillary wave and with a Wilhelmy plate for myristic acid on water for longitudinal waves. Even for the regular Laplace waves, the surface tension determined from the wavelength can be quite inaccurate, e.g., Davies and Vose [43] reported an error of $\sim 2 \mathrm{mN} / \mathrm{m}$ for hydrocarbon surfactants, and even higher for fluoroderivatives and proteins. The presence of surface active impurities is often blamed for that, and careful cleaning of the surface is required before the measurements. Suction of the outermost surface layer via a micrometer syringe is a widely used [4,43]; rinsing the surface with a movable barrier is also efficient [4]. However, meticulous cleaning does not resolve completely the problem with the low accuracy of the method. Moreover, the deviations and the low reproducibility are typical for dense surfactant monolayers, where impurities should have a minor effect. Barrier adsorption-desorption process is blamed for the negative effective surface viscosity reported by the light scattering method, but the phenomenon has never been clarified [6].

\section{Theory}

\subsection{Flat waves on a surface covered with a surfactant}

Consider a forced oscillation of the surface progressing in $x$ direction, of frequency $v$ and angular frequency $\omega=2 \pi v$. The shape of the surface is given by the equation of a progressive decaying flat wave:

$$
z^{\mathrm{S}}=a_{\mathrm{c}} \mathrm{e}^{\mathrm{i}(k x-\omega t)}=a \mathrm{e}^{-x / L_{\mathrm{d}}} \cos \left[\frac{2 \pi\left(x-x_{0}(t)\right)}{\lambda}\right] .
$$

Here, $z^{\mathrm{S}}$ is the (normal) $z$-coordinate of the surface, $k$ is the complex wave number, $\lambda=2 \pi / \operatorname{Re}(k)$ is the wavelength, $L_{\mathrm{d}}=1 / \operatorname{Im}(k)$ is the decay length, $x_{0}(t)=x_{0}(0)+\lambda v t$ is the phase, $a_{\mathrm{c}}$ is a complex amplitude and $a$ is amplitude at $x=0$. Only linear waves are considered here $(a<<\lambda)$.

The liquid (aqueous solution of octanol in our experiments) occupies the semi-infinite space $z<0$; the wavelength is significantly smaller than the depth of the trough in our 
experiment, so no correction is needed for the long wave effect (cf. ref. [34]). The wave results in oscillating velocity field in the vicinity of the surface:

$$
v_{z}=V_{z}(z) \mathrm{e}^{\mathrm{i}(k x-\omega t)}, \quad v_{x}=V_{x}(z) \mathrm{e}^{\mathrm{i}(k x-\omega t)} ;
$$

similarly, the pressure oscillates around the hydrostatic profile $p_{0}-\rho g z$,

$$
p=p_{0}-\rho g z+P(z) \mathrm{e}^{\mathrm{i}(k x-\omega t)} .
$$

The complex amplitudes $V_{z}, V_{x}$ and $P$ decay exponentially with depth [3,41]:

$$
P(z)=\xi a \frac{\rho \omega^{2}}{k} \mathrm{e}^{k z} ; \quad V_{x}(z)=a \omega\left[\xi \mathrm{e}^{k z}+(1-\xi) \frac{m}{k} \mathrm{e}^{m z}\right] ; \quad V_{z}(z)=-\mathrm{i} a \omega\left[\xi \mathrm{e}^{k z}+(1-\xi) \mathrm{e}^{m z}\right] .
$$

Here, the viscous decay number $m$ and the complex weight factor $\xi$ are related to the wave number $k$ as

$$
\begin{aligned}
& m^{2}=k^{2}-\mathrm{i} \rho \omega / \eta, \\
& \xi=1+2 \mathrm{i} \eta k^{2} / \rho \omega .
\end{aligned}
$$

Thus, at the oscillating surface of a viscous fluid, there exist two zones. The first one is thicker the amplitude decays by a factor of 2.7 for a distance of $\lambda / 2 \pi \sim 100-500 \mu \mathrm{m}$ (in the frequency range we study); in this zone, the pressure and velocity profiles do not differ much from those in an ideal fluid and the particle trajectories are circular. The second zone is of smaller thickness, $1 / \operatorname{Re}(m) \sim 25-30 \mu \mathrm{m}$, and there the amplitude of oscillations in $x$-direction is larger than the one in $z$-direction (since the ratio $m / k$ in eq. (4) is large), i.e. the particle trajectories are elliptical right next to the surface.

The mechanical wave is conjugated with oscillating material fluxes. When surfactant is present, its concentration oscillates synchronously with the wave:

$$
c=c_{0}\left[1+C(z) \mathrm{e}^{\mathrm{i}(k x-\omega t)}\right] .
$$

In addition, at the surface, the adsorption and the surface tension oscillate around their equilibrium values $\Gamma_{0}$ and $\sigma_{0}$ :

$$
\Gamma=\Gamma_{0}\left[1+a_{\Gamma} \mathrm{e}^{\mathrm{i}(k x-\omega t)}\right], \quad \sigma=\sigma_{0}-a_{\Gamma} E \mathrm{e}^{\mathrm{i}(k x-\omega t)} .
$$

Here, $a_{\Gamma}$ is the relative amplitude of the adsorption perturbation wave, and $a_{\Gamma} E$ is the amplitude of the surface tension wave; $E$ is the Gibbs elasticity of the surface. The oscillation of $\Gamma$ and $c$ is experimentally accessible for fluorescent surfactants [48]. Let the barrier adsorptiondesorption kinetics have negligible effect on the capillary wave characteristics (this is valid for octanol; for alcohols higher than decanol, we expect considerable role of the barrier adsorptiondesorption [79]). Under this assumption, local equilibrium is established between the adsorbed monolayer and the subsurface solution, and the amplitudes $C$ and $a_{\Gamma}$ are related as:

$$
C(z=0)=a_{\Gamma} E / R T \Gamma_{0},
$$

where $R$ is the gas constant. The amplitude of oscillation of the concentration varies with $z$ as

$$
C(z)=C(z=0) \mathrm{e}^{l z} \text {, where } l^{2}=k^{2}-\mathrm{i} \frac{\omega}{D} \approx-\mathrm{i} \frac{\omega}{D} .
$$

The thickness of this diffusion layer is of the order of $1 / \operatorname{Re}(l) \sim 0.5-1 \mu \mathrm{m}$ for the system we study.

The force and the mass balances at the surface determine $\xi$ (see eq. (6)), $a_{\Gamma}$, and the dispersion condition (the relation between $k$ and $\omega$ ). For soluble surfactants such as octanol, the dispersion condition of the wave corresponds to one of a viscoelastic surface: 


$$
\omega^{2}+\frac{\frac{4 \eta^{2}}{\rho^{2}} k^{2} m(k-m)-\frac{\varepsilon}{\rho} k^{3}}{1+\frac{\varepsilon}{\rho} \frac{k^{2}(k-m)}{\omega^{2}}}=\frac{\sigma}{\rho} k^{3}+g k,
$$

where $\varepsilon$ is the complex surface elasticity, given by

$$
\varepsilon=E\left(1+\mathrm{i} \frac{D c E}{R T \Gamma^{2}} \frac{l}{\omega}\right)^{-1}
$$

The diffusion term in eq. (12) becomes important at low frequency, namely $v<D c^{2} E^{2} / R^{2} T^{2} I^{4}$ ( $\sim \mathrm{Hz}$ for $0.3 \mathrm{mM}$ octanol). However, at such frequencies (gravity waves), the elasticity terms in the dispersion condition (11) are small, so the effect of the diffusion process on the wave properties is usually modest and appears in a limited range of frequencies (mostly for gravitycapillary waves). For the range we study $(v>300 \mathrm{~Hz})$, the diffusion term in eq. (12) is negligibly small and one can set $\varepsilon=E$ :

$$
\omega^{2}+\frac{\frac{4 \eta^{2}}{\rho^{2}} k^{2} m(k-m)-\frac{E}{\rho} k^{3}}{1+\frac{E}{\rho} \frac{k^{2}(k-m)}{\omega^{2}}}=\frac{\sigma}{\rho} k^{3}+g k .
$$

These results were derived by Levich [3] for complex $\omega$ rather than complex $k$ and in somewhat different, but equivalent, form. Equivalent results were obtained also by Lucassen and Hansen [80] and others. Eq. (11)\&(13) have been challenged many times [43,81], based on disagreement with experimental results from the surface relaxational spectrometers ${ }^{1}$. For the monolayer we study, however, eq. (13) appears to be accurate, see sec. 4.3.

Approximate solutions to eq. (13) can be easily derived in two limiting cases - completely mobile and completely immobile surface.

For completely mobile surface (no surfactant), elasticity is negligible, $E=0$, and eq. (13) simplifies to

$$
\omega^{2}+\frac{4 \eta^{2}}{\rho^{2}} k^{2} m(k-m)=\frac{\sigma}{\rho} k^{3}+g k .
$$

For real $\omega$ (forced wave that does not decay in time), the dispersion relation (14) has seven solutions overall. Either one or two of these are physical and fulfil the conditions $\operatorname{Re}(k)>0$, $\operatorname{Im}(k)>0$ and $\operatorname{Re}(m)>0$ - these correspond to the Laplace and the Marangoni wave modes. The real part of eq. (14) is almost unaffected by the viscosity term, i.e. Kelvin's equation for $\lambda$ [1] is approximately correct (deviations appear at $v<\rho \sigma^{2} / \eta^{3}$ and $\lambda>\eta^{2} / \sigma \rho$, i.e. optical frequencies for water but waves in the $\mathrm{kHz}$ for viscous fluids like glycerol). Moreover, for the frequencies we study, $g k$ is relatively small compared to $\sigma k^{3} / \rho$; in addition, for the Laplace mode, it holds true that $\operatorname{Re}(m) \approx-\operatorname{Im}(m)>>\operatorname{Re}(k) \gg>\operatorname{Im}(k)$. This leads to the following explicit solution to eq. (14)\&(5):

$$
\begin{aligned}
& m=(1-\mathrm{i}) \sqrt{\frac{\rho}{2 \eta} \omega} \\
& \lambda=2 \pi\left(\frac{\sigma}{\rho}\right)^{1 / 3} \omega^{-2 / 3}
\end{aligned}
$$

\footnotetext{
${ }^{1}$ Eq. (13) is a source of confusion for yet another reason: the first classical work of Levich [3] contains a typo which persists in some secondary sources. The other important work in the field, ref. [80], uses a wave form with negative $\omega$ (corresponding to wave propagating backwards), which has also been a source of errors.
} 


$$
L_{\mathrm{d} 0}=3 \sigma / 4 \eta \omega .
$$

The last formula was derived by Stokes [2]. The index 0 in $L_{\mathrm{d} 0}$ stands for neat surface (no surfactant).

The limiting case of a completely immobile surface corresponds to $E \rightarrow \infty$. If we use eq. (5) and neglect the $g k$ term, at $E \rightarrow \infty$ we can simplify the imaginary part of eq. (13) to

$$
\frac{\eta \omega}{\sigma} \operatorname{Re}[k(k+m)]=\operatorname{Im}\left(k^{3}\right)
$$

where for $m$ and $\lambda$ the approximations (15) and (16) still hold. Solving this equation for $L_{\mathrm{d}}=1 / \operatorname{Im}(k)$ yields

$$
L_{\mathrm{d} \infty}=\frac{6 R e^{+1 / 2}-1}{1+R e^{-1 / 2}} \times \frac{\lambda}{2 \pi},
$$

where the dimensionless group $R e$ is the Reynolds number for the wave (cf. [82]),

$$
R e=\sqrt{\frac{\sigma \rho \lambda}{8 \pi \eta^{2}}},
$$

with characteristic velocity $\lambda v \sim(\sigma / \rho \lambda)^{1 / 2}$. The value of $R e$ is of the order of 50 for the system we study.

The general case. As the elasticity increases from 0 to $\infty$, the decay length $L_{\mathrm{d}}$ changes from Stokes' value $L_{\mathrm{d} 0}$ for a fully fluid surface, eq. (17), to $L_{\mathrm{d} \infty}$ of the completely solidified one, eq. (19). However, the function $L_{\mathrm{d}}(E)$ passes through a pronounced minimum, as widely discussed in the literature [4,3]. For aqueous systems, this minimum appears around $E \sim 10 \mathrm{mN} / \mathrm{m}$. Moreover, there is little dependence of $L_{\mathrm{d}}$ on $E$ for $E>50 \mathrm{mN} / \mathrm{m}$ and below $E<1 \mathrm{mN} / \mathrm{m}$, so this is the range of values of $E$ that can be measured using the decay length of the Laplace waves. The current practice for extraction of the viscoelastic properties of the surface from the data for $\lambda$ and $L_{\mathrm{d}}$ is to solve numerically the dispersion condition (13) (or a variant of it). This is cumbersome because the dispersion equation has multiple solutions (two physical and many unphysical), and the dimensionless groups in the equation vary by orders of magnitude as the frequency changes, which might produce numerical problems.

An approximated solution is useful with such problems. One such can be obtained by expanding the elasticity term in eq. (13) in series with respect to the large parameter $2 \pi L_{\mathrm{d}} / \lambda$ (and then proceed in a manner similar to the derivation of eq. (19) from eq. (18)). The derivation is straightforward but involved; the result reads:

$$
\frac{2 \pi L_{\mathrm{d}}}{\lambda}=\operatorname{Re} \frac{\left[\begin{array}{c}
12 e^{4} R e^{5 / 2}-2 e^{3}(13 e+12) R e^{2}+4 e^{2}\left(7 e^{2}+13 e+6\right) R e^{3 / 2} \\
-e\left(44 e^{2}+43 e+12\right) R e+\left(32 e^{2}+15 e+3\right) R e^{1 / 2}-8 e
\end{array}\right]}{\left[\begin{array}{c}
2 e^{4} R e^{3}-2 e^{3}(e+1) R e^{5 / 2}-e^{2}(2 e-1) R e^{2} \\
+8 e^{2}(e+1) R e^{3 / 2}-2 e(7 e+3) R e+2(4 e+1) R e^{1 / 2}-1
\end{array}\right]} .
$$

Here, $e=E / \sigma$ is the dimensionless elasticity (0-3 for most surfactant monolayers), and $R e$ is the Reynolds number given by eq. (20). The error of this approximation is less than $3 \%$ (Figure 2).

Kelvin's formula (16) is actually inaccurate for surfaces with surfactant, which can be important given the high experimental precision for the wavelength achievable with modern surface wave apparatuses. A more accurate result can be obtained by solving iteratively the real part of eq. (13) for $\lambda$. If Kelvin's wavelength is treated as the zeroth approximation $\lambda_{0}$, we can substitute $\lambda$ for $\lambda_{0}$ in the small terms in eq. (13) - namely, the real part of the elasticity term and $g k$. The elasticity term is then again expanded in series at $2 \pi L_{\mathrm{d}} / \lambda_{0} \rightarrow \infty$; leaving the leading term of this expansion is sufficiently accurate. The large term $\operatorname{Re}\left(\sigma k^{3} / \rho\right)$ is approximated as $8 \pi^{3} / \lambda_{0}^{3} \times\left[1-3\left(\lambda-\lambda_{0}\right) / \lambda_{0}\right]-6 \pi / L_{\mathrm{d}}^{2} \lambda_{0}$. Solving eq. (13) thus approximated leads to 


$$
\frac{\lambda-\lambda_{0}}{\lambda_{0}}=-\frac{e^{2} R e^{2}-e(e+1) R e^{3 / 2}+2 e R e-2 e R e^{1 / 2}+(e+1)}{3 R e^{3 / 2}\left[2 e^{2} \operatorname{Re}-2 e(e+1) R e^{1 / 2}+(e+1)^{2}\right]}-\left(\frac{2 \pi L_{\mathrm{d}}}{\lambda_{0}}\right)^{2}+\frac{g \rho^{1 / 3}}{3 \omega^{4 / 3} \sigma^{1 / 3}} .
$$

The correction is significant - a monolayer elasticity of $5-50 \mathrm{mN} / \mathrm{m}$ alters the wavelength by, e.g., $20 \mu \mathrm{m}$; for comparison, a surface tension increase of $3 \mathrm{mN} / \mathrm{m}$ will have the same effect. Similar analytic approximations have been obtained by Henderson and Rajan [82]. The dependence of this correction on the concentration of a surfactant is illustrated in Figure $2 b$ on the example of aqueous octanol solutions.
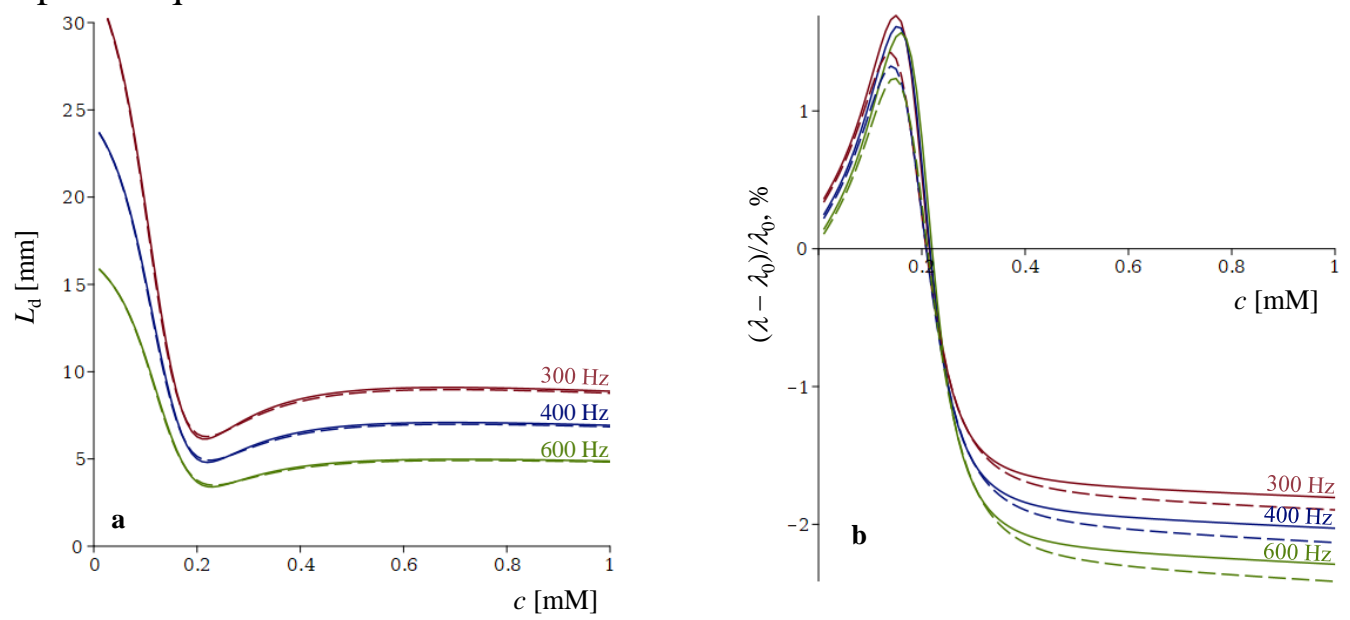

Figure 2. Decay length (a) and correction of Kelvin's equation for the wavelength (b) as a function of the concentration of octanol in water at three frequencies. Solid lines: exact solution to the dispersion condition (13); dashed lines: the approximate solutions (21)\&(22).

The surface tension and the elasticity are computed from the sticky disc model at each concentration, see SI1.

Waves decaying with time. In the dispersion relation (13), we assumed that $k$ is a complex wavenumber $\left(=2 \pi / \lambda+\mathrm{i} / L_{\mathrm{d}}\right)$, while $\omega$ is a real angular frequency. The equation has the exact same form for real wavenumber $(=2 \pi / \lambda$, a space sinusoid $)$ and a complex frequency ( $\omega=2 \pi v-\mathrm{i} / t_{\mathrm{d}}$, where $t_{\mathrm{d}}$ is the decay time). If $k=\mathrm{f}(\omega)$ is the same dispersion function then one can write for each of these cases:

(time-decaying wave)

$$
\begin{aligned}
& \frac{2 \pi}{\lambda_{\text {time-decaying }}}=\mathrm{f}\left(2 \pi v-\frac{\mathrm{i}}{t_{\mathrm{d}}}\right) ; \\
& \frac{2 \pi}{\lambda_{\text {space-decaying }}}+\frac{\mathrm{i}}{L_{\mathrm{d}}}=\mathrm{f}(2 \pi v) .
\end{aligned}
$$

(space-decaying wave)

For waves of decay length $L_{\mathrm{d}}$ much longer than $\lambda$ and decay time much longer than the wave period, the first relation (23) can be expanded into series with respect to $t_{\mathrm{d}}$, then $\mathrm{f}(2 \pi v)$ can be substituted according to eq. (24), and finally $\mathrm{df} /\left.\mathrm{d} \omega\right|_{\omega=2 \pi \nu}$ can be expanded in series with respect to $L_{\mathrm{d}}$. The result reads:

$$
\begin{gathered}
\frac{2 \pi}{\lambda_{\text {time-decaying }}} \approx f(2 \pi v)-\left.\frac{\mathrm{df}}{\mathrm{d} \omega}\right|_{\omega=2 \pi v} \frac{\mathrm{i}}{t_{\mathrm{d}}} \approx \frac{2 \pi}{\lambda_{\text {space-decaying }}}+\frac{\mathrm{i}}{L_{\mathrm{d}}}+\frac{1}{\lambda_{\text {space-decaying }}^{2}} \frac{\mathrm{d} \lambda_{\text {space-decaying }}}{\mathrm{d} v} \frac{\mathrm{i}}{t_{\mathrm{d}}}, \\
\text { i.e. } \lambda_{\text {time-decaying }} \approx \lambda_{\text {space-decaying }} \equiv \lambda \text { and } \frac{L_{\mathrm{d}}}{t_{\mathrm{d}}} \approx-\lambda^{2} \frac{\mathrm{d} v}{\mathrm{~d} \lambda}=V_{\mathrm{g}} .
\end{gathered}
$$

Thus, the decay length and the decay time are related through the group velocity $V_{\mathrm{g}}$ of the wave [5]; moreover, the wavelengths of the space-decaying and time-decaying waves are approximately the same. It must be kept in mind that these relations are valid approximations 
for the Laplace mode waves, but not for the fast-decaying Marangoni mode. Even for the Laplace waves, and especially close to the damping maximum, both relations (25) are inaccurate. It should be also noted that the hydrodynamic problem allows a whole family of waves that decay both in space and time, i.e. proportional to

$$
\exp [\mathrm{i}(k x-\omega t)]=\cos \left[2 \pi\left(\frac{x}{\lambda}-v t\right)\right] \exp \left(-\frac{x}{L_{\mathrm{d}}}-\frac{t}{t_{\mathrm{d}}}\right) .
$$

The decay length of such waves varies with the decay time as $1 / L_{\mathrm{d}}=1 / L_{\mathrm{d} \text {,space }}-1 /\left(V_{\mathrm{g}} t_{\mathrm{d}}\right)$. Thus, a source oscillating with amplitude that decays with time (with $t_{\mathrm{d}}$ between $t_{\mathrm{d} \text {,time }}$ and $\infty$ ) will excite a wave that has a decay length somewhere between $L_{\mathrm{d} \text {,space }}$ and $\infty$.

Recent developments. The theory of capillary waves has made significant progress in the last two decades. Particularly important are the advances in the field of non-linear waves, which were reviewed in ref. [40]; the recent works on the theory of thermal capillary waves has been discussed in ref. [6]. The main theoretical results for the dispersion relationship of ripples from before 1996 have been summarized in the detailed review by Noskov [4]. Here, the work on ripples from after 1996 is considered.

Rajan and Henderson $[82,83]$ generalized several previous theories to obtain the dispersion relation for a wave at the interface between two Newtonian liquids covered with an elastic monolayer of an insoluble surfactant. They also did the painstaking work of giving approximate solutions of the type of eq. (21)-(22) to the dispersion equation. Rajan [42] extended this work to another important case - a flat film of a fluid between two other fluids.

One relatively new development is the analysis of the dispersion condition for viscoelastic media such as gels and biological soft matter. Onodera and Choi [84] investigated the waves on the surface of an elastic material. They showed that the elastic Rayleigh mode exists as a surface wave only at low frequencies. Above a certain critical frequency $\left(\omega_{\mathrm{cr}}=G^{3 / 2} / \rho^{1 / 2} \sigma\right.$, where $G$ is the shear-elastic constant), the Rayleigh wave is no longer localized at the interface and instead extends to the whole bulk of the elastic material. As for the capillary modes, it was shown that they induce the emission of diverging elastic waves normal to the surface which dissipate the energy of the capillary wave, i.e. the capillary waves are also not true surface waves anymore. Onodera and Choi accordingly introduced the term pseudo-surface or leaky surface capillary waves. If the damping is not strong ( $G$ is small) these waves can still be detected. The dispersion equation they follow is

$$
\omega^{2}=\frac{\sigma}{\rho} k^{3}+\frac{4 G}{\rho} k^{2}
$$

Thus, short waves of this type approach Kelvin's dispersion relation, while long waves are elastic dispersionless (as $\omega \sim k$ ) and appear as a second Rayleigh mode. Chantelot et al. [49] investigated the waves at the surface of a gel layer of finite thickness. They showed that the confined thickness results in occurrence of multiple modes at low frequencies, both theoretically and experimentally. Kappler, Netz, and Zendehrouud [85,86] analysed interfaces of inherent surface viscoelasticity (i.e. with surfactant) between viscoelastic media (including compressible fluids, Kelvin-Voigt materials, and Maxwell materials).

Waves on cylindrical jets and spherical drops have not been investigated in the same detail as flat waves, despite the great potential for application of surface relaxational spectroscopy on jets and droplets as a control tool in inkjet printing [19,20,21,22]. The surface elasticity has been introduced only relatively recently to the dispersion condition of jet waves by Noskov [1], who derived an equation that corrects Bohr's formula [87] used for the determination of the surface tension from the wave characteristics. Another important field of investigation are waves on charged surfaces, jets and droplets, which have important role in electrojetting (see, e.g., $[28,29])$. 


\section{An optical capillary wave apparatus}

\subsection{Materials and methods}

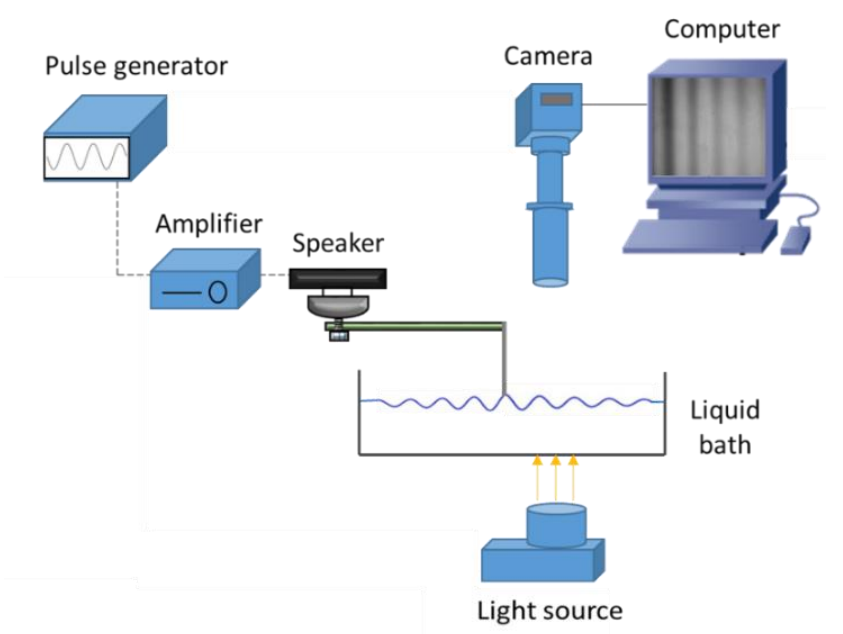

Figure 3. Schematic of the experimental configuration.

To characterize capillary waves, we built our own apparatus and developed a code to process the wave images captured in the experiment.

Experimental setup. For our in-build experimental setup, mechanical generation was chosen as a method for wave excitation, for the purpose of versatility (see sec. 2.2). Optical visualization of the wave profile in space with a high-speed video camera was utilized as a detection method. This approach has not been used previously for the purpose of relaxational spectroscopy of fluid interfaces, see sec. 2.3. Using the entire profile of the wave (as opposed to point methods) allows large amount of data to be gathered quickly. Flat waves were studied since the images of flat symmetry allow for faster methods of analysis.

A schematic of the experimental setup is shown in Figure 3. The studied liquid, $2 \mathrm{~cm}$ deep, was contained in a petri dish, $20 \mathrm{~cm}$ in diameter. A glass slide, of size $50 \times 30 \times 0.8 \mathrm{~mm}^{3}$, was used to perturb the liquid surface near the centre of the petri dish to create the capillary waves. With these dimensions in place, the petri dish wall was distant enough to avoid a reflected wave to interfere with the capillary waves next to the glass slide; interaction of the wave with the meniscus at the wall was also avoided. One side of the glass slide was slightly immersed into the liquid; the other side was attached to a loud speaker (VISATON, EX60S) through a horizontal strip. The actuation slide-speaker system allowed horizontal and vertical displacement by a triaxial translation stage to change the glass slide immersion depth. The speaker acted as an exciter moving vertically up and down and was driven by a sinusoidal voltage signal created by a pulse generator (TG2511A, TTi Ltd, UK) and an amplifier (Marantz, PM6010OSE) to control both the amplitude and the frequency of the waves.

To detect the wave profile, a high-speed video camera (Photron, Fastcam Mini AX200) was placed perpendicular to the liquid surface close to the glass slide, as shown in Figure 3. Careful rectangulation of the waves was required - the wave propagation direction must be at an angle as close to zero as possible with respect to the $x$ coordinate of the image. The photographed area was as close to the plate as the shade from the plate allowed (at larger distances, deviation from flat wave geometry should be expected). The camera was equipped with an optical magnification lens (EC74687, 4.5X) and was used to acquire images of $8 \times 4.5 \mathrm{~mm}^{2}$ field of visualization, with spatial resolution of $8 \mu \mathrm{m} /$ pixel. Frames of different phase were selected for the following analysis. A cool light source (SCHOTT, MLEP-A070W1LR) was used to provide uniform lighting to back-illuminate the system. 


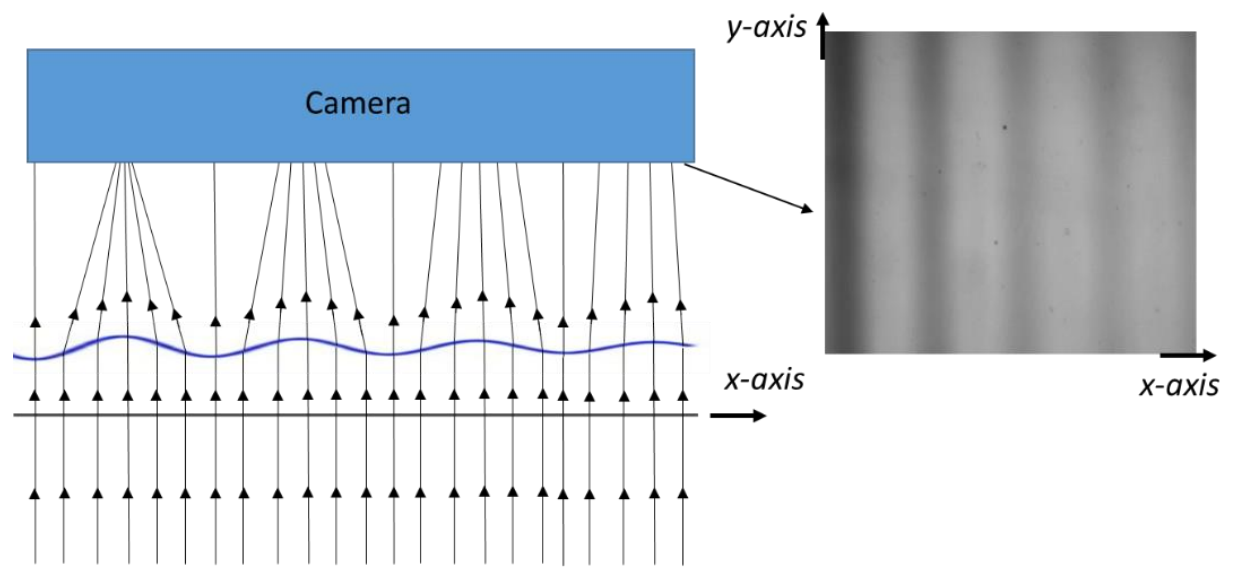

Figure 4. Reflection of light from the wavy surface. (Multimedia view)

The light rays from the light source reaches the camera after crossing the wavy liquid surface. The crests and troughs of the wave act as convex and concave lenses, respectively $[9,59]$. The camera records an image of grey stripes (see Figure 4 multimedia view), with dark grey corresponding to troughs. For small amplitude of the waves, the location of the camera is well below the focal point of all wave crests (cf. ref. [9] where it is the opposite); as a result, the grey value is a linear function of the curvature of the surface. To reconstruct the wave profile, the mean grey value as a function of the distance $x$ to the source was analysed. Only relative amplitude was obtained in this way, which is sufficient to determine $\lambda$ and $L_{\mathrm{d}}$ with high precision. Our equipment allows, in principle, to measure accurate absolute amplitude of the capillary wave, via variation of the distance between the camera and the surface (as in ref. [9,59]), but this was of no interest for the questions investigated in this work. We limited the studied range of frequencies between 300 and $500 \mathrm{~Hz}$. However, direct tests showed that range from 100 to $650 \mathrm{~Hz}$ is easily achieved with our apparatus.

Materials. To test the method, $0.255 \mathrm{mM}$ solution of 1-octanol was used (Sigma-Aldrich, $99 \%$ ) in deionized water, prepared and studied at $22 \pm 1{ }^{\circ} \mathrm{C}$. There are several reasons for choosing this system. Octanol is a well-studied surfactant for which surface tension data are available $[88,89,90,91]$ and the equation of state is parametrized [92], i.e. $\sigma, \Gamma$ and $E$ are known with reasonable accuracy from literature. Moreover, the elasticity was measured directly by the oscillating bubble method [93], allowing for some comparison. Octanol is of low volatility (the evaporation of hexanol can affect the wave properties significantly). The barrier resistance for adsorption-desorption can be neglected [4] (for higher alcohols, the flip-flop process can be expected to affect the viscoelastic properties of the monolayer [79]). The chosen concentration corresponds to coverage of $\sim 3.3 \mu \mathrm{mol} / \mathrm{m}^{2}$, which is about $50 \%$ of the collapse value $(\sim 6.8$ $\mu \mathrm{mol} / \mathrm{m}^{2}$ for normal alkanols). This makes sure that the octanol dominates the surface, and adventitious surface-active impurities (which always complicate pure water experiments) will have a minor effect. Our reason to work at concentration of $0.255 \mathrm{mM}$ was that there the conditions are close to maximum damping (see Figure 2), i.e. to $\mathrm{d} L_{\mathrm{d}} / \mathrm{d} E=0$. This is the worstcase scenario for the sensitivity of the measured $L_{\mathrm{d}}$ data to the value of the elasticity, so it allows the limiting sensitivity of the method to be determined. Preliminary experiments with other concentrations were done until the conditions of the experiment were optimized, but the results for this concentration are enough to specify the parameters of the tool we have built. 


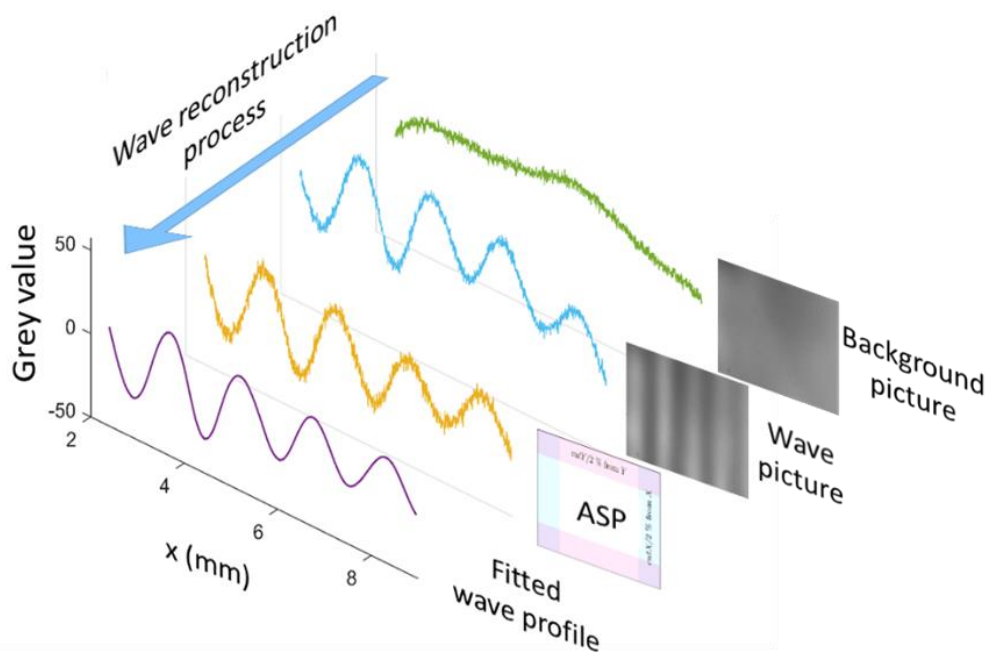

Figure 5. Wave reconstruction process. The mean background grey value profile is subtracted from the mean grey value profile of the wavy surface, to obtain the averaged-subtracted profile (ASP). The wavelength and the decay length are obtained from ASP via regression.

Data processing. An algorithm was developed to collect the grey value data from the captured images, to reconstruct the wave profiles, to compare them with eq. (1) and to determine the wavelength and the decay length via regression. Additional description of the code is provided in SI3 and in ref. [94]. The wave reconstruction process is illustrated in Figure 5. The main steps of the procedure are outlined below.

(i) At each frequency, the code operates with $N$ images of a wavy surface (we operated with $N=10-40$ ), together with one image of the background. Each image is a square matrix of grey values (the images we used were $912 \times 512$ pixels). The wave travels in $x$-direction.

(ii) To minimize the background noise, the grey values of the image of the wavy surface and the background image are subtracted, to produce $N$ respective subtracted pictures. Two methods of subtraction were tested; the first is the direct one,

$$
z^{\mathrm{S}}=K_{1}\left(I-I_{\text {background }}\right),
$$

where $K_{1}$ [m/grey unit] is an arbitrary proportionality constant. The second one assumes the following relationship between the second derivative of the wave shape and the intensity:

$$
z_{x x}^{\mathrm{S}}=K\left(I_{\text {background }}-I\right) / I \quad(\equiv \Delta I / I) .
$$

a formula that follows from the optical theory of the problem [59]; $K$ is in $\mathrm{m}^{-1}$. Derivation is given in SI2. Direct tests showed that the two options (28)\&(29) are nearly equivalent; we present below only the results from eq. (29), as it highlights the optical nature of the method (the grey value is proportional to the curvature of the surface rather than the absolute amplitude).

(iii) Next, the procedure averages the values of $\Delta I / I$ along each horizontal line (i.e. for all $y=1-512$ for our images). This produces $N$ sets of data for $\Delta I / I$ vs. $x$ (averaged-subtracted profiles, ASP).

(iv) Each set of $\Delta I / I$ vs. $x$ data is fitted with the formula

$$
\frac{\Delta I}{I}=a_{0}+a_{I} \mathrm{e}^{-x / L_{\mathrm{d}}} \cos \left[\frac{2 \pi\left(x-x_{0}\right)}{\lambda}\right],
$$

a combination of eq. (1) and (29) where a constant correction $a_{0}$ is introduced. For perfect perturbation of the background image by the wave, the constant $a_{0}$ should be equal to zero; we instead observed homogeneous alteration of the mean grey value (the wavy images tended to be darker than the background).

We compared different regression methods to process the image data (modified Newton, Sequential Quadratic Programming, Simplex). We found that the computation time and the 
reliability were dependent less on the method and more on the initial approximation for the regression parameters $\left(a_{0}, a, x_{0}, \lambda\right.$ and $\left.L_{\mathrm{d}}\right)$. Therefore, we developed a procedure that determines a good initial approximation, see SI3. The regression in the final Maple code was performed using Maple's default modified Newton method implemented in the LSSolve function [95].

(v) Next, the regression parameters, the frequency and the standard deviation of the model and the data were exported to a spreadsheet where the data were filtered. Two filters were applied; the first one was based on the dispersion (if a datum had dispersion $20 \%$ higher than the minimum in a given run and given frequency, it was deleted). The second one was based on the $L_{\mathrm{d}}$ value - if it was outside the interval (mean $\left.L_{\mathrm{d}}\right) \pm 50 \%$, the respective datum was deleted. The second filter was applied as, in certain images, non-linear wave effects appeared, probably due to imperfect oscillation of the plate, see below. The value of $L_{\mathrm{d}}$ was found to be particularly sensitive to this effect.

The code allows $\sim 40$ images to be processed per minute on a standard desktop computer. Around $25 \%$ of the images (usually the noisy ones) could not be fitted with the code and were filtered out. A second version of the code was run for all those images, which used the results from one fit in a run as an initial approximation for the rest in the same run. With this additional step, only $10 \%$ of the images had to be filtered out. In general, the additional step is not necessary as the images lost due to filtering can be compensated for simply by using more images.

\subsection{Optimization of the experimental parameters}

The light intensity has to be carefully adjusted for optimal precision of the method. Low intensity of light results in inadequate contrast and grey value variation of the order of the background noise. On the other hand, high intensity produces non-linear transfer function amplitude $\rightarrow$ grey value, due to grey value approaching saturation (255 units) in the vicinity of the lightest spots. Figure 6 shows an example where the grey value resolution increases with intensity until a limit is reached above which the maxima appear flattened due to the light saturation.

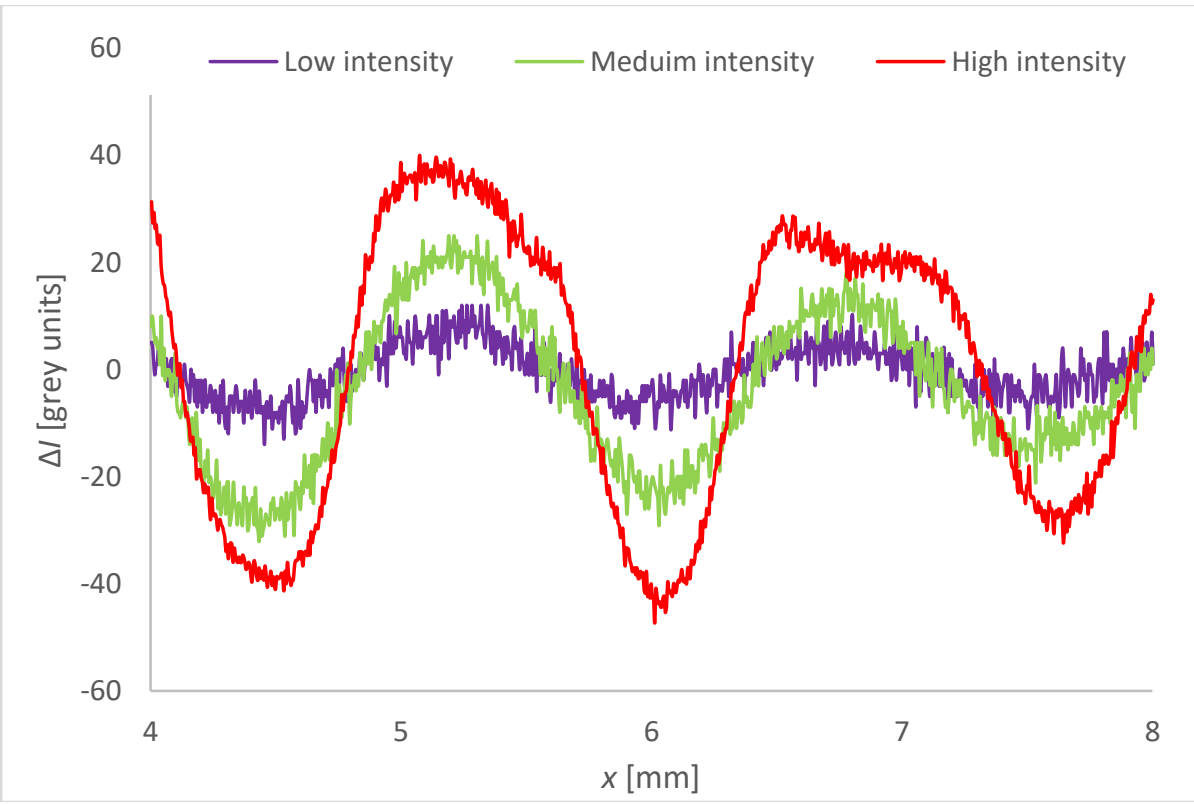

Figure 6. Effect of the intensity of the light source on the observed wave profile: mean grey value $\Delta I$ of an averaged-subtracted wave profile at three intensities of illumination

$$
\text { at } v=300 \mathrm{~Hz} \text {. }
$$


Wave amplitude. The excitation system (the glass slide and the horizontal strip) has natural frequencies, which can be calculated from the cantilever beam natural frequency formula [96],

$$
f_{n}=\frac{K_{n}}{2 \pi} \sqrt{\frac{E_{\mathrm{c}} I_{\mathrm{c}} g}{w l_{\mathrm{s}}^{4}}} ;
$$

here $E_{\mathrm{c}}$ is the modulus of elasticity of the cantilever, $I_{\mathrm{c}}$ is the moment of inertia, $l_{\mathrm{s}}$ is the strip length, $w$ is the load per unit length, and $K_{n}$ represent a constant referring to the mode of vibration. When the excitation frequency of the speaker approaches a natural frequency of the system, resonance appears; the resulting high amplitude improves the resolution but it can cause chaotic disturbance, undesired complex waves, and grey value approaching saturation. This can be avoided by reducing the voltage of the speaker input signal. On the other hand, when the excitation frequency is far from the natural frequencies, the wave amplitude becomes too small to be detected by the visualization system. The initial amplitude can be increased by escalating the speaker input voltage but this is limited to the maximum power input that the speaker can handle. We preferred another approach to increase the amplitude when needed - by altering the immersion depth and angle of the slide.

Slide immersion depth and angle. We used ultra-thin glass slides under inclination angle of $\theta=90^{\circ}, 105^{\circ}$ and $120^{\circ}$. At angles higher than $120^{\circ}$, complex waves were excited, perhaps due the large liquid contact area with the slide side; therefore, we did not study such angles. The immersion depth of the slide edge in pure water was varied from $d=0$ to $4 \mathrm{~mm}$ (beyond 4 $\mathrm{mm}$, the wave amplitude became independent of $d$ ).

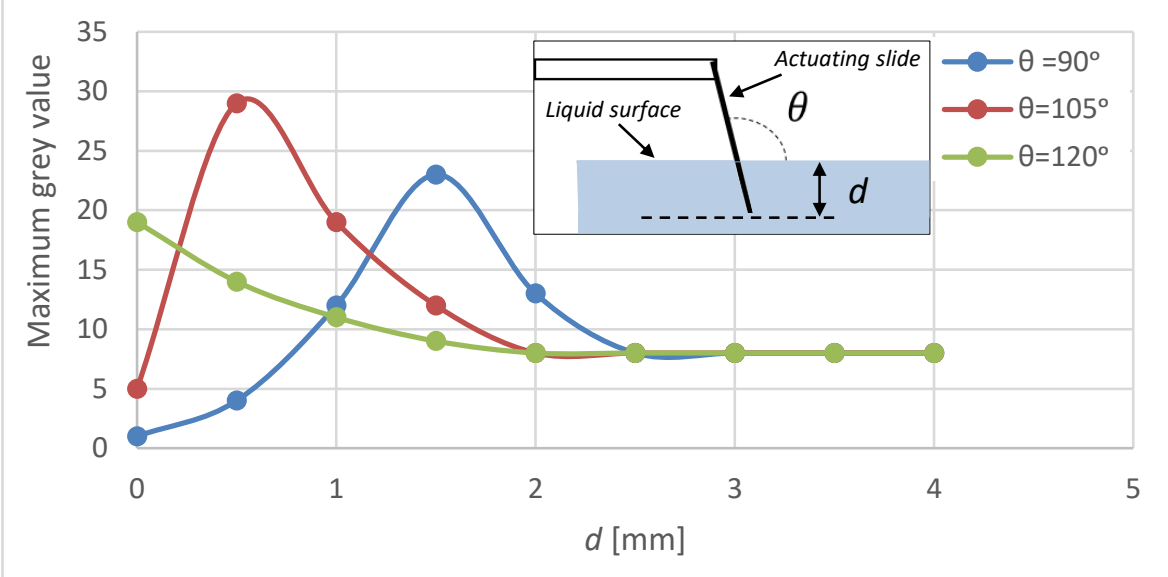

Figure 7. Effect of the glass slide angle and the immersion depth on the wave amplitude. The mean grey value in the maximum nearest to $3.7 \mathrm{~mm}$ is plotted. The definition of inclination angel $(\theta)$ and immersion depth $(d)$ are shown in the inset.

The results in Figure 7 show the effect of the immersion depth $d$ on the wave amplitude at a distance of $3.7 \mathrm{~mm}$ away from the glass slide. For plate normal to the surface $\left(\theta=90^{\circ}\right)$, the amplitude increases with the immersion depth until it reaches a turning point at $1.5 \mathrm{~mm}$ depth above which the amplitude starts to decrease. Increasing the angle to $105^{\circ}$ leads to an increased peak value at lower immersion depth at $0.5 \mathrm{~mm}$. This turning point corresponds to the optimum $d$ and $\theta$ values to maximize the amplitude for frequencies far from the natural frequencies of the actuation system, and most experiments were performed with this configuration. By increasing the angle $\theta$ further to $120^{\circ}$, the turning point disappear and the amplitude monotonously decreases with depth. 


\subsection{Wavelength and decay length}

In this section, the results from three runs of the apparatus are analysed. The runs were with slight variation in conditions (different observation field, varied amplitude). A programme of frequencies was investigated in each run. The frequency range between 300 and $500 \mathrm{~Hz}$ was studied.

We observed a significant variation of the noise with frequency: for example, all wave images at $360 \mathrm{~Hz}$ were significantly less noisy than those at $500 \mathrm{~Hz}$, see Figure 8. Unexpectedly, the noisiness of the images did not correlate strongly with the standard deviation of $\lambda$ and $L_{\mathrm{d}}$ determined from a set of images at a single frequency. Thus, the values of $L_{\mathrm{d}}$ determined from the noisy images at 420 and $500 \mathrm{~Hz}$ were as reproducible as those for the smooth images at 300 and $360 \mathrm{~Hz}$. The main source of noise was most probably overtone vibrations of the mechanical unit near the resonance frequencies, see eq. (31). In certain configurations (high power and large distance between the surface and the camera), non-linear relationship between grey value intensity and wave amplitude was observed, i.e. the approximated linearized relationship (29) became invalid. Such cases correspond to large wave amplitude, where the location of the camera approaches the focal point of the crests. The mild non-linear deviations from eq. (29) appear as higher maxima and shallower minima. Upon further increase in the wave amplitude, each crest produces two parallel grey value peaks separated by a shallow grey value minimum, and then caustics appear. These effects can be used to determine the absolute amplitude of the wave $[9,59]$, but this is beyond the scope of this work. We only dealt with images in the linear region.
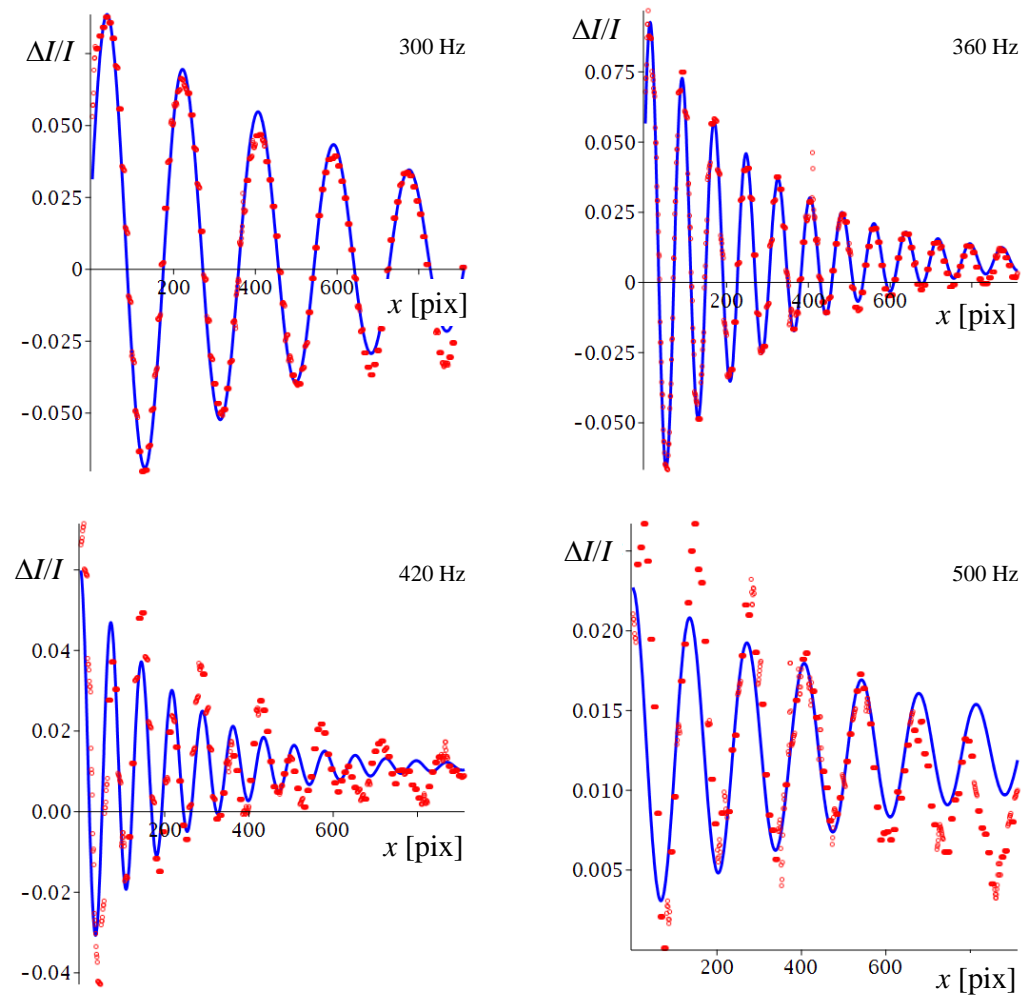

Figure 8. Sample averaged-subtracted profiles at several frequencies. Points are relative grey value intensity, $\left(I_{0}-I\right) / I$, obtained from a single image after averaging along the $y$-axis. The first two examples are for smooth, and the next two are for noisy images. Calibration varies (18.182 and $8.7336 \mu \mathrm{m} / \mathrm{pixel})$. 
The results for the wavelength and the decay length as functions of the frequency are summarized in Table 1 and Figure 9. The relative standard deviation in $\lambda$ is $\pm 0.5 \%$ on the average; the decay length $L_{\mathrm{d}}$ is determined with standard deviation of around $\pm 7 \%$. Overall, the optimal conditions for photographing the wave vary with frequency; moreover, the optimal conditions are different for measuring $L_{\mathrm{d}}$ and $\lambda$. Best results for $L_{\mathrm{d}}$ are obtained where the observation field is around $2 \times L_{\mathrm{d}}$ in length ( $\sim 5-10 \mathrm{~mm}$, depending on the frequency); for the low frequencies, the standard deviations of $L_{\mathrm{d}}$ increase because this condition is not fulfilled. Best results for the wavelength are obtained for observation field of length $3-5 \times \lambda=3-6 \mathrm{~mm}$.

Table 1. Measured wavelength and decay length vs. frequency of forced flat waves at the surface of $0.255 \mathrm{mM}$ solution of octanol.

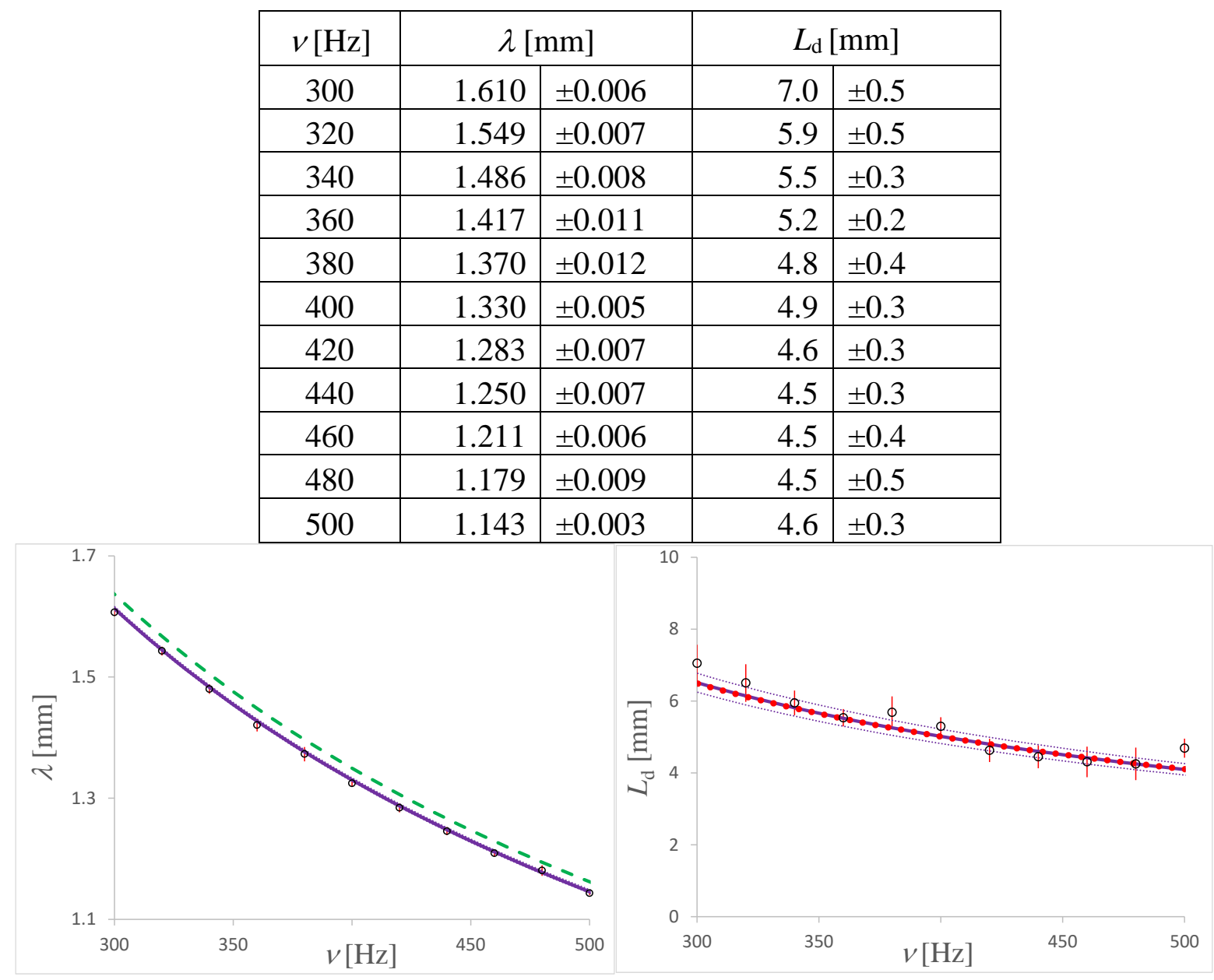

Figure 9. Wavelength and decay length of waves at the surface of $0.255 \mathrm{mM}$ aqueous octanol.

Points: measured data, see Table 1. Solid lines: theory, eq. (13) with $\sigma=62.0 \mathrm{mN} / \mathrm{m}$ (measured independently) and $E=10.6 \mathrm{mN} / \mathrm{m}$ (fitted value). To indicate the sensitivity to the surface elasticity, the shift of the theoretical line for $E=10.6 \pm 1 \mathrm{mN} / \mathrm{m}$ is indicated in the second plot. Dashed line: wavelength calculated via Kelvin's equation.

The final question is how to use the measured dependences of $\lambda$ and $L_{\mathrm{d}}$ vs. $v$ to determine the properties of the surface. For the system we study, all parameters of eq. (13) are known, i.e. the dispersion relation is formally a predictive equation. However, there is significant uncertainty in both surface characteristics involved, the surface tension $\sigma$ and the surface elasticity $E$. 
We measured the surface tension of the studied solution directly, $\sigma=62.0 \mathrm{mN} / \mathrm{m}$, using the pendant drop method. An image of a pendant drop was taken using a CCD camera (uEye UI-2230-C, IDS) with a microscopic lens (Optem Zoom 125) and processed using ImageJ software to calculate the surface tension. The pendant drop was kept inside enclosed transparent cell to avoid any evaporation; five minutes were allowed to reach equilibrium. The measurement was done at $22{ }^{\circ} \mathrm{C}$. The measured value $\sigma=62.0 \mathrm{mN} / \mathrm{m}$ can be compared with literature data. At octanol concentration of $0.255 \mathrm{mM}$, the variation in $\sigma$ reported in the literature is between 62 and $66 \mathrm{mN} / \mathrm{m}$ (see SI1). A third value can be obtained from Ivanov's sticky disc adsorption model parametrized for the adsorption of the whole homologous series of alkanols at water|air [92]; this model yields $\sigma=65 \mathrm{mN} / \mathrm{m}$ (see SI1 for details). Both the measured and the computed value of $\sigma$ fall in the range of values reported in the literature. The sticky disc model was further used to compute also the adsorption $\Gamma$ and the surface elasticity; the results are $\Gamma=3.3 \mu \mathrm{mol} / \mathrm{m}^{2}$ and $E=10.5 \mathrm{mN} / \mathrm{m}$. The error in $E$ can be expected to be similar to that in $\sigma$, i.e. $\pm 2 \mathrm{mN} / \mathrm{m}$. For comparison, for $0.3 \mathrm{mM}$ octanol, Wantke et al. [93] determined $E=9 \mathrm{mN} / \mathrm{m}$ from tensiometric data, and $E=13 \mathrm{mN} / \mathrm{m}$ using the oscillating bubble method. Finally, for the density and viscosity of the solution at $22{ }^{\circ} \mathrm{C}$, the following values were used: $\rho=997.8-1.2=996.6 \mathrm{~kg} / \mathrm{m}^{3}\left(1.2 \mathrm{~kg} / \mathrm{m}^{3}\right.$ is correction for the density of air $)$ and $\eta=0.9573 \mathrm{mPa} \cdot \mathrm{s}$.

The simplest way to extract the elasticity from the capillary wave measurements is by fitting the data for $L_{\mathrm{d}}$ vs. $v$ with the approximate eq. (21), with eq. (20) and (16) substituted in it. This yields good regression (Figure 9); the best fitting value of the Gibbs elasticity is $E=10.3 \pm 1.1 \mathrm{mN} / \mathrm{m}$, in excellent agreement with the value from the sticky disc model (see Table 2).

However, Kelvin's equation (16) is not a particularly good approximation for the wavelength, because the elasticity $E$ has a significant effect on $\lambda$, see eq. (22). For example, at $300 \mathrm{~Hz}$, the exact wavelength that follows from the dispersion equation (13) with $\sigma=62.0$ and $E=10.5 \mathrm{mN} / \mathrm{m}$ is $\lambda=1.613 \mathrm{~mm}$; in comparison, if the elasticity is ignored, one obtains $\lambda=1.637 \mathrm{~mm}$. The experimental wavelength is $1.610 \pm 0.006 \mathrm{~mm}$. This means that the effect of $E$ on $\lambda$ (which is nearly always ignored in the literature [34,43]) is actually significantly higher than our experimental uncertainty: from the values above, the effect of the elasticity on $\lambda$ is $1613-1637=-24 \mu \mathrm{m}$, while the experimental standard deviation of $\lambda$ is $\pm 6 \mu \mathrm{m}$. Gravity also has non-negligible effect on $\lambda(+6 \mu \mathrm{m}$ at $300 \mathrm{~Hz})$ and has to be accounted for. In comparison, the diffusion correction in the dispersion condition (11)-(12) has effect smaller than the uncertainty limits: the diffusion coefficient of octanol is $7 \times 10^{-10} \mathrm{~m}^{2} / \mathrm{s}$ [79], and taking it into account leads to increase in the theoretical values of $\lambda$ by $\sim 2 \mu \mathrm{m}$ and of $L_{\mathrm{d}}$ by $\sim 0.02 \mathrm{~mm}$ in the studied frequency range.

Therefore, a more accurate approach for the determination of $E$ from Laplace wave characterization would take into account the data for both $\lambda$ and $L_{\mathrm{d}}$. To demonstrate that, we constructed the following objective function for the regression:

$$
\Delta^{2}(E)=\frac{\sum\left(\lambda_{\mathrm{th}}\left(v_{i} ; E\right)-\lambda_{i}\right)^{2}}{\delta_{\lambda}^{2}}+\frac{\sum\left(L_{\mathrm{d}, \mathrm{h}}\left(v_{i} ; E\right)-L_{\mathrm{d}, i}\right)^{2}}{\delta_{L_{\mathrm{d}}}^{2}} .
$$

The weight factors $\delta_{\lambda}$ and $\delta_{L \mathrm{~d}}$ were set equal to the mean experimental standard deviations from Table 1, 0.007 and $0.37 \mathrm{~mm}$. The theoretical values $\lambda_{\text {th }}$ and $L_{\mathrm{d} \text {,th }}$ were computed in two ways. The first is from the exact dispersion equation (13); all seven solutions were found using the default Maple routine for complex polynomial equation, and the correct Laplace mode solution was identified as the physical solution of longer $L_{\mathrm{d}}$. The minimum of $\Delta^{2}$ was found at $E=10.6 \pm 0.9 \mathrm{mN} / \mathrm{m}$, i.e. the use of the data for $\lambda$ decreased the uncertainty in $E$ by $20 \%$. The 
second way to compute $\lambda_{\text {th }}$ and $L_{\mathrm{d}, \mathrm{th}}$ is through the approximate solutions (21)\&(22). The result is the same, and the optimization procedure is faster by 2-3 orders of magnitude. However, the first approach has the benefit of being easily generalized to more complicated dispersion equations (e.g., diffusion must be accounted for at $v<100 \mathrm{~Hz}$ ).

Finally, we tested how accurate would a capillary wave method be for determination of the surface tension $\sigma$ of surfactant systems. We minimized the function (32) with respect to two parameters, $E$ and $\sigma$ (rather than fixing $\sigma$ to the value $62.0 \mathrm{mN} / \mathrm{m}$ measured with the pendant droplet method). The optimization yields best values $\sigma=61.7 \mathrm{mN} / \mathrm{m}$ and $E=10.4 \mathrm{mN} / \mathrm{m}$. The uncertainty in $\sigma$ is around $\pm 0.3 \mathrm{mN} / \mathrm{m}$ (compared to $\pm 0.2 \mathrm{mN} / \mathrm{m}$ for pendant droplet); however, using two parameters instead of just one increases the uncertainty in $E$, and we see the capillary wave apparatus most of all as a method to measure elasticity.

The results from the regressions are compared to literature data for $\sigma$ and $E$ of octanol in Table 2.

Table 2. Comparison of the measured surface tension and elasticity to literature data.

\begin{tabular}{|r|c|c|c|}
\hline & $\begin{array}{c}\text { literature/theoretical } \\
\text { values }\end{array}$ & $\begin{array}{c}\text { one-parametric } \\
\text { regression }\end{array}$ & $\begin{array}{c}\text { two-parametric } \\
\text { regression }\end{array}$ \\
\hline$C$ & $0.255 \mathrm{mM}$ & & $61.7 \pm 0.25 \mathrm{mN} / \mathrm{m}$ \\
\hline$\sigma$ & $\begin{array}{r}{ }^{\mathrm{a}} 62.0 \mathrm{mN} / \mathrm{m} \\
{ }^{\mathrm{b}} 62 \ldots 66 \mathrm{mN} / \mathrm{m} \\
{ }^{\mathrm{c}} 65 \mathrm{mN} / \mathrm{m}\end{array}$ & fixed to $^{\mathrm{a}} 62.0 \mathrm{mN} / \mathrm{m}$ & $10.4 \pm 1.2 \mathrm{mN} / \mathrm{m}$ \\
\hline$E$ & ${ }^{\mathrm{c}} 10.5 \mathrm{mN} / \mathrm{m}$ & $10.6 \pm 1.0 \mathrm{mN} / \mathrm{m}$ & \\
\hline$\Gamma$ & ${ }^{\mathrm{c}} 3.3 \mu \mathrm{mol} / \mathrm{m}^{2}$ & & \\
\hline
\end{tabular}

a Surface tension measured directly with a pendant droplet. ${ }^{\mathrm{b}}$ Published tensiometric data $[88,89,90,91] .{ }^{c}$ Computed from Ivanov's sticky disc adsorption model [92] (see SI1).

\section{Conclusion}

We constructed a surface wave apparatus that combines a high-speed camera with a mechanical oscillator, to measure the wavelength and decay length of flat Laplace waves. The detection method is suitable for small-amplitude linear capillary waves of frequency in the range $10^{2}-10^{3} \mathrm{~Hz}$. The main advantages of the technique are:

(i) simple construction and inexpensive;

(ii) high precision ( $\pm 7 \mu \mathrm{m}$ or $0.5 \%$ for $\lambda$, and $\pm 0.4 \mathrm{~mm}$ or $7 \%$ for $L_{\mathrm{d}}$ );

(iii) quick procedure for extraction of $\lambda$ and $L_{\mathrm{d}}$ from the images.

We demonstrated that, for octanol solution of known $\sigma$ and $E$, the apparatus gives both $\lambda$ and $L_{\mathrm{d}}$ equal to the theoretical values, within the experimental uncertainty.

Compared to other methods, it seems that only very few works report higher precision in the determination of $\lambda$ and $L_{\mathrm{d}}$ than what we achieved. Shmyrov et al. [34], who also used a 3D reconstruction technique, reported data with $0.5 \%$ error in $\lambda$, and $1 \%$ in $L_{\mathrm{d}}$ for pure liquid, but this increased to $1 \%$ and $5 \%$, respectively, for waves damped by surfactant [18]. Our apparatus seems to be of similar precision to those in Ref. $[4,8,43]$ and better than many others (error of $\pm 50 \%$ is common for $L_{\mathrm{d}}$ [46]). The wave data obtained with our apparatus allowed determination of the surface elasticity $E$ with precision of $\pm 0.9 \mathrm{mN} / \mathrm{m}$ for the test system we investigated, which is a good result compared to alternative methods [93]. This makes the capillary wave technique a useful source of data for the equation of state of monolayers for both 
soluble and insoluble surfactants. Surface elasticity data are particularly sensitive to the lateral attraction parameter $\beta$ [98], and allow surface phase transitions to be detected, which could be otherwise difficult for soluble monolayers [99]. The apparatus allowed surface tension to be simultaneously measured, with precision of $\pm 0.3 \mathrm{mN} / \mathrm{m}$. We showed that the surface elasticity affects significantly the wavelength (rather than just the decay length) of the capillary wave, to well above the experimental precision. The disregard of the effect of $E$ on $\lambda$ is the reason for the low accuracy of surface tension determined from the wavelength data reported in the literature $( \pm 1-2 \mathrm{mN} / \mathrm{m}$ when surfactant is present, compared to $\pm 0.3 \mathrm{mN} / \mathrm{m}$ for neat surfaces $[34,43]$ when Kelvin's equation is used without correction for $E$ ).

Possible improvements and range of applications. It is possible to achieve significantly better precision of $\lambda, L_{\mathrm{d}}$ and $E$ determined with the technique developed. To do so, one can

(i) use more images. Currently, we use 10 images at most frequencies, $512 \times 912$ pixels. Using 100-1000 is easy and will improve the precision of the instrument dramatically.

(ii) Better control of the temperature is needed for a high-precision instrument (currently, $T$ is constant within $\pm 1^{\circ} \mathrm{C}$ ).

(iii) Better elasticity values will be obtained if wider frequency range is studied (100-1000 Hz should present no difficulty).

(iv) Better control of the edge effects (especially for longer waves, deviations from flat symmetry appear).

The simplicity of our equipment means that the method is very flexible. With appropriate processing codes, we can study waves decaying in time (not just in space); waves in thin liquid films on a transparent solid substrate or on a liquid; waves of cylindrical symmetry from a point source (as in ref. [34]); waves for monolayers for which the imaginary part of the complex elasticity is significant (i.e. the adsorption-desorption rates can be investigated). The apparatus can be used also to study the effect of evaporation on the dispersion equation under controlled conditions.

Supplementary materials: Surface tension isotherm of octanol (Section SI1); Light refraction by a flat wave (Section SI2); Code for processing the camera images (Section SI3).

Acknowledgements. We would like to thank our students Abdulkadir Abdulkadir and Hussain Taleouine for doing some of the experiments and data processing, and Dr Rafa Castrejón-Pita for helpful discussions.

\section{Nomenclature}

a wave amplitude

$c \quad$ surfactant concentration

$D \quad$ bulk diffusion coefficient

$E \quad$ surface Gibbs elasticity, $E=-\Gamma \mathrm{d} \sigma / \mathrm{d} \Gamma$

$e$ dimensionless elasticity, $e=E / \sigma$

$G \quad$ shear-elastic constant

I grey value intensity

$I_{0} \quad$ grey value intensity of the background image

i imaginary unit

$k \quad$ complex wave number

$l \quad$ diffusion layer decay number, eq. (10)

$m \quad$ viscous decay number, eq. (5)

$k_{\mathrm{B}} \quad$ Boltzmann constant 


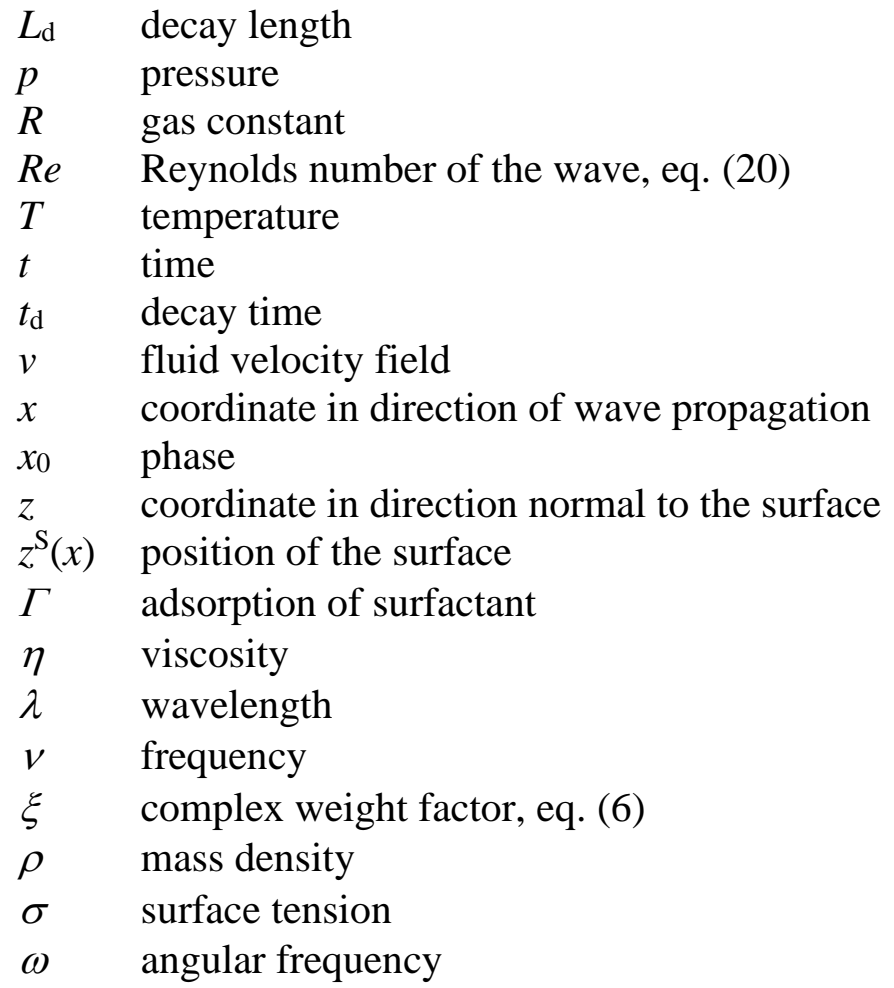

\section{References}

1. W. Thomson (Lord Kelvin), Phyl. Trans. 64 (1871) 445 (Phil. Mag. 42 (1871) 368).

2. G. G. Stokes, Mathematical and Physical Papers, Vol. 3 (Cambridge U. P., Cambridge, 1901), pp. 1-141.

3. V. Levich. Physicochemical hydrodynamics. Moscow, 1959.

4. B.A. Noskov. Fast adsorption at the liquid-gas interface. Adv. Colloid Interface Sci. 69 (1996) 63-129.

5. E.H. Lucassen-Reynders, J. Lucassen. Properties of capillary waves. Adv. Colloid Interface Sci. 2 (1969) 347-395.

6. D. Langevin. Light scattering by liquid surfaces, new developments. Adv. Colloid Interface Sci. 289 (2021) 102368.

7. M.G. Munoz, F. Monroy, P. Hernandez, F. Ortega, R.G. Rubio, D. Langevin. Langmuir 19 (2003) 2147-2154.

8. M. Safouane, D. Langevin. Surface viscoelasticity of concentrated salt solutions: specific ion effects. ChemPhysChem 10 (2009) 222-225.

9. J. Garrett, Marine Research 25 (1967) 279-291.

10. I. Sergievskaya, S. Ermakov, T. Lazareva, J. Guo. Marine Pollution Bulletin 146 (2019) 206-214.

11. L. Krutyansky, A. Brysev, F. Zoueshtiagh, P. Pernod, D. Makalkin. Measurements of interfacial tension coefficient using excitation of progressive capillary waves by radiation pressure of ultrasound in microgravity. Microgravity Sci. Technol. 31 (2019) 723-732.

12. Y.M. Lau, J. Westerweel, W. van de Water, Langmuir 36 (2020) 5872-5879.

13. R.S. Hansen, R. Adin Mann. Propagation characteristics of capillary ripples. J. Appl. Phys. 35 (1964) 152-158. 
14. K.Y. Lee, T. Chou, D.S. Chung, E. Mazur. Direct measurement of the spatial damping of capillary waves at liquid-vapor interfaces. J. Phys. Chem. 97 (1993) 12876-12878.

15. C.H. Sohl, K. Miyano, J.R. Ketterson. Rev. Sci. Instrum. 49 (1978) 1464-1469.

16. F. Behroozi, B. Lambert, B. Buhrow. Noninvasive measurement of viscosity from damping of capillary waves. ISA Transactions 42 (2003) 3-8.

17. F. Behroozi, J. Smith, W. Evan. Stokes' dream: measurement of fluid viscosity from the attenuation of capillary waves. Am. J. Phys. 78 (2010) 1165-1169.

18. A. Shmyrov, A. Mizev, I. Mizeva, A. Shmyrova. Electrostatic precipitation of exhaled particles for tensiometric examination of pulmonary surfactant. J. Aerosol Sci. 151 (2021) 105622.

19. N. Morita, A.A. Khalate, A.M. van Buul, H. Wijshoff. Inkjet printheads, ch. 3 in: S.D. Hoath, ed. Fundamentals of inkjet printing. Willey, 2016.

20. S.D. Hoath, W.-K. Hsiao, G.D. Martin, S. Jung, S.A. Butler, N.F. Morrison, O.G. Harlen, L.S. Yang, C.D. Bain, I.M. Hutchings. Oscillations of aqueous PEDOT:PSS fluid droplets and the properties of complex fluids in drop-on-demand inkjet printing. J. Non-Newtonian Fluid Mech 223 (2015) 28-36.

21. H.J.J. Staat, A. van der Bos, M. van der Berg, H. Reinten, H. Wijshoff, M. Versluis, D. Lohse. Ultrafast imaging method to measure surface tension and viscosity of inkjetprinted droplets in flight. Exp. Fluids 58 (2017) 2.

22. L Yang, L.J. Adamson, C.D. Bain. Study of liquid jet instability by confocal microscopy. Rev. Sci. Instrum. 83 (2012) 073104.

23. S.D. Hoath, ed. Fundamentals of inkjet printing. Willey, 2016, ch. 1-2.

24. P. Chu, J. Finch, G. Bournival, S. Ata, C. Hamlett, R.J. Pugh. A review of bubble breakup. Adv. Colloid Interface Sci. 270 (2019) 108-122.

25. A. Scheludko. Sur certaines particularit é s des lames mousseuses. Proc. Konikl. Ned. Acad. Wet. B65 (1962) 86-99.

26. A. Vrij. Possible mechanism for the spontaneous rupture of thin free liquid films. Discuss. Faraday Soc. 42 (1966) 23-33.

27. I.B. Ivanov, B. Radoev, E. Manev, A. Scheludko. Theory of the critical thickness of rupture of thin liquid films. Trans. Faraday Soc. 66 (1970) 1262-1273

28. A. Said Ismail, J. Yao, H.H. Xia, J.M. Lopez-Herrera, and J.P.W Stark. Breakup length of electrified liquid jets: Scaling laws and applications. Phys. Rev. Applied 10 (2018) 064010.

29. H.H. Xia, A. Said Ismail, J. Yao, J.P.W. Stark, Scaling Laws for Transition from Varicose to Whipping Instabilities in Electrohydrodynamic Jetting, Phys. Rev. Applied 12 (2019) 014031.

30. J. Israelashvili, Intermolecular and surface forces, $3^{\text {rd }}$ ed. Elsevier, 2011.

31. R.F. Kayser. Effect of capillary waves on surface tension. Phys. Rev. A 33 (1986) 19481956.

32. R. Slavchov, V. Dutschk, G. Heinrich, B. Radoev. Justification of biexponential rate law of spreading over heterogeneous and rough surface. Colloids Surf. A 354 (2010) 252-260.

33. E. Raphaël, P.-G. de Gennes, Capillary gravity waves caused by a moving disturbance: wave resistance. Phys. Rev. E 53 (1996) 3448-3455.

34. A. Shmyrov, A. Mizev, A. Shmyrova, I. Mizeva. Capillary wave method: an alternative approach to wave excitation and to wave profile reconstruction. Phys. Fluids 31 (2019) 012101.

35. I. Langmuir, Surface motion of water induced by wind, Science 87 (1938) 2250. 
36. E.J. Bock, J. Adin Mann. On ripple dynamics II. A corrected dispersion relation for surface waves in the presence of surface elasticity. J. Colloid Interface Sci. 129 (1989) 501-505.

37. K. Miyano, B. M. Abraham, L. Ting, D. T. Wasan. Longitudinal surface waves for the study of dynamic properties of surfactant systems I. Instrumentation. J. Colloid Interface Sci. 92 (1983) 297-302.

38. L. Ting, D. T. Wasan, K. Miyano, S.-Q. Xu. Longitudinal surface waves for the study of dynamic properties of surfactant systems II. Air-solution interface. JCIS 102 (1984) 248-259.

39. C. Lemaire, D. Langevin. Longitudinal surface waves at liquid interfaces: measurement of monolayer viscoelasticity. Colloids and surfaces 65 (1992) 101-112.

40. E. Falcon, N. Mordant. Experiments in Surface Gravity-Capillary Wave Turbulence. Annual Review of Fluid Mechanics, Annual Reviews, In press, 54, pp.1 - 27. 10.1146/annurev-fluid-021021-102043. hal-03279480.

41. H. Lamb. Hydrodynamics. Cambridge University Press, 1932, Ch. 9.

42. G.K. Rajan. A three-fluid model for the dissipation of interfacial capillary-gravity waves. Phys. Fluids 32 (2020) 122121.

43. J.T. Davies, R.W. Vose. On the damping of capillary waves by surface films. Proceedings of the Royal Society of London. Series A, Mathematical and Physical Sciences 286 (1965) 218-234.

44. N.A. Vinnichenko, A.V. Pushtaev, Y.Y. Plaksina, A.V. Uvarov. Measurements of liquid surface relief with moon-glade background oriented Schlieren technique. Exp. Therm. Fluid Sci. 114 (2020) 110051.

45. D. Thiessen, A. Scheludko, Kolloid-Zeitschrift und Zeitschrift für Polymere 218 (1967) 139-148.

46. J.R. Saylor, A.J. Szeri, G.P. Foulks, Experiments in fluids 29 (2000) 509-518.

47. X. Shao, C.T. Gabbard, J.B. Bostwick, J.R. Saylor. On the role of meniscus geometry in capillary wave generation. Experiments in Fluids 62, 1\{4. issn: 14321114. https://doi.org/10.1007/s00348-021-03161-3 (2021).

48. S.L. Strickland, M. Shearer, K.E. Daniels. J. Fluid Mech. 777 (2015) 523-543.

49. P. Chantelot, L. Domino, and A. Eddi, How capillarity a_ects the propagation of elastic waves in soft gels, Physical Review E 101 (2020) 032609.

50. C. Stenvot, D. Langevin, Langmuir 4 (1988) 1179-83.

51. R.J. Skarlupka, H. Yu, K. Ito, B.B. Sauer, M. Sano, Dynamic interfacial properties of poly(ethylene oxide) and polystyrene at toluene/water interface. Langmuir 6 (1990) 1379-1388.

52. J. Novev, N. Panchev, R.I. Slavchov. Chem. Eng. Sci. 171 (2017) 520-533.

53. E.P. Furlani, B.G. Price, G. Hawkins, A.G. Lopez, Thermally induced Marangoni instability of liquid micro-jets with application to continuous inkjet printing. Proceedings of NSTI-Nanotechnology Conference 2 (2006) 534-537.

54. D.S. Subbaramaiya. Proc. Ind. Acad. Sci. (1937) 333-339.

55. K. Ito, B.B. Sauer, R.J. Skarlupka, M. Sano, H. Yu, Langmuir 6 (1990) 1379-1388.

56. B. Issenmann, C. Laroche, E. Falcon. Wave turbulence in a two-layer fluid: coupling between free surface and interface waves. Europhys. Lett. 116 (2016) 64005.

57. A. Cazaubiel, F. Haudin, E. Falcon, M. Berhanu. Forced three-wave interactions of gravitycapillary surface waves. Phys. Rev. Fluids 4 (2019) 074803.

58. L. Deike, M. Berhanu, E. Falcon. Decay of capillary wave turbulence. Phys. Rev. E 85 (2012) 066311.

59. A.H. Shooley, The ripple-tank as an aid to phase-front visualization. Rep. US Nav. Research Lab. 3559. 
60. F. Moisy, M. Rabaud, K. Salsac. A synthetic Schlieren method for the measurement of the topography of a liquid interface. Exp. Fluids 46 (2009) 1021-1036.

61. J. Kolaas, B.H. Riise, K. Sveen, A. Jensen. Bichromatic synthetic Schlieren applied to surface wave measurements. Exp. Fluids 59 (2018) 128.

62. A. Przadka, B. Cabane, V. Pagneux, A. Maurel, P. Petitjeans. Fourier transform profilometry for water waves: How to achieve clean water attenuation with diffusive reflection at the water surface? Exp. Fluids 52 (2012) 519-527.

63. S. Wildeman. Real-time quantitative Schlieren imaging by fast Fourier demodulation of a checkered backdrop. Exp. Fluids 59 (2018) 97

64. F. Leckler, F. Ardhuin, C. Peureux, A. Benetazzo, F. Bergamasco, V. Dulov. Analysis and interpretation of frequency-wavenumber spectra of young wind waves. J. Phys. Ocean 45 (2015) 2484-2496.

65. S.L. Strickland, M. Shearer, K.E. Daniels. J. Fluid Mech. 777 (2015) 523-543.

66. M. Berhanu, E. Falcon. Space-time resolved capillary wave turbulence. Phys. Rev. E 87 (2013) 033003.

67. I. Mizeva. Capillary wave-detection algorithm based on cylindrical solitary waves. J. Phys.: Conf. Ser. 1945 (2021) 012037.

68. X. D. Shi, M. P. Brenner, and S. R. Nagel. A cascade of structure in a drop falling from a faucet. Science 0036-8075 265, 219 (1994).

69. D. M. Henderson, W. G. Pritchard, and L. B. Smolka. On the pinch-off of a pendant drop of viscous fluid. Phys. Fluids 1070-6631, https://doi.org/10.1063/1.869435 9, 3188 (1997).

70. T. A. Kowalewski. On the separation of droplets from a liquid jet. Fluid Dyn. Res. 0169-5983 https://doi.org/10.1016/0169-5983(95)00028-3 17, 121 (1996).

71. H. Dong, W. W. Carr, and J. F. Morris. An experimental study of drop-on-demand drop formation," Phys. Fluids 18, 072102 (2006).

72. F. Wang, F. P. Contò, N. Naz, J. R. Castrejón-Pita, A. A. Castrejón-Pita, C. G. Bailey, W. Wang, J. J. Feng, and Y. Sui, A fate-alternating transitional regime in contracting liquid filaments, J. Fluid Mech. 860, 640 (2019).

73. T. Yamada, K. Sakai. Observation of collision and oscillation of micro-droplets with extremely large shear deformation. Phys. Fluids, 24 (2) (2012) 022103.

74. A.S. Mohamed, J.M. Lopez-Herrera, M.A. Herrada, L.B. Modesto-Lopez, A.M. Gañán-Calvo, Effect of a Surrounding Liquid Environment on the Electrical Disruption of Pendant Droplets. Langmuir 32 (2016) 6815-6824.

75. K.S. Kwon, An inkjet vision measurement technique for high frequency jetting. Rev. Sci. Instrum. 85 (2014) 065101.

76. J. Rayleigh, On the instability of a cylinder of viscous liquid under capillary forces, Philos. Mag. 34 (1892) 145-154.

77. F.J. García, H. González, F.J. Gómez-Aguilar, A.A. Castrejón-Pita, J.R. Castrejón-Pita. Evolution of Gaussian wave packets in capillary jets. Phys. Rev. E. 100 (2019) 053111.

78. H. González, J. Arcenegui, F.J. García de Bollullos, J.R. Castrejón-Pita, and A.A. Castrejón-Pita. Self-Stimulated Capillary Jet. Phys. Rev. Applied 15 (2021) 014054.

79. I. Minkov, D. Arabadzieva, E. Mileva, R.I. Slavchov, Soft Matter 15 (2019) 1730-1746.

80. J. Lucassen, R.S. Hansen, J. Colloid Interface Sci. 23 (1967) 319-328.

81. F.C. Goodrich, J. Phys. Chem. 66 (1962) 1858-1863.

82. G.K. Rajan, D.M. Henderson. Linera waves at a surfactant-contaminated interface separating two fluids: dispersion and dissipation of capillary-gravity waves. Physics of fluids 30 (2018) 072104.

83. G.K. Rajan, Dissipation of interfacial Marangoni waves and their resonance with capillary-gravity waves, Int. J. Eng. Sci. 154 (2020) 103340.

84. Y. Onodera and P.-K. Choi, Surface-wave modes on soft gels, The Journal of the Acoustical Society of America 104 (1998) 3358-3363. 
85. J. Kappler, R.R. Netz. Multiple surface wave solutions on linear viscoelastic media. EPL 112 (2015) 19002.

86. S. Zendehrouud, R.R. Netz, J. Kappler. Linear waves at viscoelastic interfaces between viscoelastic media. Phys. Rev. Fluids (2021), submitted.

87. N. Bohr, Determination of the surface-tension of water by the method of jet vibration. Phil. Trans. Royal. Soc. A 209 (1909) 281-317

88. V.B. Fainerman, S.V. Lylyk, Koll. Zh. 45 (1983) 500.

89. J.R. Hommelen, J. Colloid Science 14 (1959) 385.

90. M. Aratono, S. Uryu, Y. Hayami, K. Motomura, R. Matuura, J. Colloid Interface Sci. 98 (1984) 33.

91. W. Barzyk, K. Lunkenheimer, P. Warszyński, B. Jachimska, A. Pomianowski. Colloids Surf. A 443 (2014) 515-524.

92. R. Slavchov, I. Ivanov, Soft Matter 13 (2017) 8829-8848.

93. K.-D. Wantke, H. Fruhner, J. Fang, K. Lunkenheimer. J. Colloid Interface Sci. 208 (1998) 34-48.

94. R. Slavchov, B. Peychev, A. Said Ismail (2021). Processing code for optical characterization of flat capillary waves [Software]. Deposited on Queen Mary Research Online, https://doi.org/10.17636/10173212.

95. Maple 2020. Maplesoft, a division of Waterloo Maple Inc., Waterloo, Ontario.

96. W.C. Young, R.G. Budynas. Roark's Formulas for Stress and Strain. 7nd Edition, McGraw-Hill, Chapter 16 (2002) 767-768.

97. M.W. Kim, D.S. Cannell, Phys. Rev. A 13 (1976) 411.

98. R. Slavchov, I.B. Ivanov, J. Colloid Interface Sci. 532 (2018) 746-757

99. B. Peychev, R.I. Slavchov. J. Colloid Interface Sci. 594 (2021) 372-388 